\title{
How Cheap Talk Enhances Efficiency in Threshold Public Goods
}

\section{Games* $^{*}$}

\author{
Thomas Palfrey $^{\dagger} \quad$ Howard Rosenthal ${ }^{\ddagger} \quad$ Nilanjan Roy ${ }^{\S}$
}

June 10, 2015

\begin{abstract}
This paper uses a Bayesian mechanism design approach to investigate the effects of communication in a threshold public goods game. Individuals have private information about contribution costs. Individuals can each make a discrete contribution. If the number of contributors is at least equal to the threshold, a public benefit accrues to all members of the group. We experimentally implement three different communication structures prior to the decision move: (a) simultaneous exchange of binary messages, (b) larger finite numerical message space and (c) unrestricted text chat. We obtain theoretical bounds on the efficiency gains that are obtainable under these different communication structures. In an experiment with three person groups and a threshold of two, we observe significant efficiency gains only with the richest of these communication structures, where participants engage in unrestricted text chatting. In that case, the efficiency bounds implied by mechanism design theory are achieved.
\end{abstract}

JEL Classification Numbers: C72, C92, D82, D83, H41

Keywords: Communication, cheap talk, message space, public good provision

\footnotetext{
${ }^{*}$ We are grateful for comments from seminar audiences at several universities and conferences. We acknowledge the financial support of grants from the National Science Foundation (SES-0962802 and SES-1426560), the Gordon and Betty Moore Foundation, and the Russell Sage Foundation. Kirill Pogorelskiy provided excellent research assistance. We especially thank John Ledyard for valuable comments on the theoretical section that extends earlier joint work by Ledyard and Palfrey. The usual caveat applies.

${ }^{\dagger}$ California Institute of Technology

${ }^{\ddagger}$ New York University

${ }^{\S}$ City University of Hong Kong
}

(C) 2015. This manuscript version is made available under the Elsevier user license http://www.elsevier.com/open-access/userlicense/1.0/ 


\section{Introduction}

We investigate how communication influences public good provision in a threshold public goods game with private information about contribution costs. The provision of threshold public goods combines free riding incentives with a coordination problem, both of which are further complicated if there is private information. Pre-play communication between agents provides a potential path to overcoming these problems, but whether communication is effective in practice, and how its effectiveness depends on the structure of communication and private information, are questions that remain largely unanswered both theoretically or empirically. This paper makes three contributions to addressing these questions. First, by modeling the game with communication as a Bayesian mechanism design problem, we are able to develop some theoretical bounds on the gains that can be attained from different pre-play communication protocols. Second, we show how these bounds depend on the distribution of private information and on the communication structure - in particular the richness of the message space. Third, we design and conduct an experiment where we vary both the communication structure and the distribution of private information.

We present several results. First, in the experiment we find that communication has significant beneficial effects only when the group members communicate in natural language. Restricting subjects to coarser message spaces, such as a binary message space or to one-time reports of their private information partially solves the coordination problem, but not enough to produce a statistically significant improvement compared with groups that were not allowed to communicate. A second finding is that the effectiveness of pre-play communication depends on the distribution of private information. In half of our data, it was common knowledge that all subjects had contribution costs that were less than or equal to the benefit of the public good, implying that it was common knowledge that, for every subject, it is optimal to contribute if contribution is pivotal for the provision of the public good. In the other half of the data, the distribution of contribution costs was such that its support included costs that exceeded the benefit, and hence it was common knowledge that any group member with such a high cost has a dominant strategy to free ride. In this second variant, natural language communication was much less effective and helped only after substantial experience was gained. This sharp difference in the effect of communication is also reflected in the theoretical bounds implied by the optimal mechanism. A binding individual rationality constraint, which is present only in the high cost treatment, sharply reduces the amount of public good provision that can be supported. Thus, we establish both theoretically and behaviorally, that the effectiveness of private communication depends on both the richness of the message space and the distribution of private information.

To keep the analysis and experimental design simple, players in the threshold public goods game 
have only a binary choice - to contribute or not, and the public good is produced if and only if at least some threshold number of group members choose to contribute. This class of games includes the social dilemmas studied by Dawes et al. (1986), Offerman et al. (1998), and Palfrey and Rosenthal (1991), and shares similar strategic elements to the volunteer's dilemma, entry games, and participation games studied by Goeree and Holt (2005). Contributions are non-refundable, so that an efficient outcome requires that exactly the threshold number of contributions are made. Too many contributions or too few contributions reflect coordination failure. Because the group members have different contribution costs, (ex ante) efficient provision also requires that the contributions are made only by the lowest cost members of the group. Thus heterogeneity of contribution costs create a second kind of coordination problem, and private information exacerbates this latter coordination problem with an incentive compatibility problem. In all cases there is a free rider problem, in the sense that any contributor would prefer to switch roles with any non-contributor, regardless of their contribution costs.

The three forms of pre-play communication we consider were carefully chosen. The coarsest message space we consider is binary. ${ }^{1}$ In the communication stage with binary messages, each group member announces an "intention" to either contribute or not, which is then followed by a simultaneousmove contribution stage with binding decisions, so the communication stage can be viewed as a direct signal about contribution in the final stage of the game or alternatively as a "practice game", where one's first round contribution decision has no direct payoff consequences. The second message space is somewhat richer, where group members simultaneously announce any number in the support of the distribution of contribution costs, thus mimicking a direct mechanism (but without a mediator), which is then followed by a contribution stage with binding decisions. The richest communication structure we consider is natural language communication where the communication stage consists of a fixed time period during which chat messages can be broadcast continuously among the group members. After the chat stage, binding contribution decisions are made simultaneously by all group members. In addition to varying the structure of communication, we varied the distribution of contribution costs. In the " $C=1$ " condition, it is always incentive compatible for the two lowest cost individuals to contribute and thus provide the public good. In the " $C=1.5$ " condition, incentive compatibility problems could result in the good not being provided even though provision was socially optimal.

The remainder of the paper is organized as follows. The related literature is reviewed in Section 2. Section 3 specifies the experimental design, theoretical framework and the central hypotheses. Section 4 presents the experimental results and analysis. The last section concludes.

\footnotetext{
${ }^{1} \mathrm{~A}$ binary message space was also explored in past work (Palfrey and Rosenthal (1991)).
} 


\section{Related Literature}

\section{Theory}

Selfish players may choose to reveal private information through costless and non-binding communication or cheap talk; such revelation can lead to efficiency gains, as shown by Crawford and Sobel (1982). The problem has been formulated in generality by Forges (1986) and Myerson (1986). Palfrey and Rosenthal (1991) were the first to investigate the effects of cheap talk in a model where players have private information about costs to contribute towards the provision of a public good. They considered a 'threshold public good game' where provision requires contributions from at least a minimum number of people. Using a binary communication setting, they showed that perfect coordination is not Bayesian-incentive compatible and that players have weak incentives to free-ride in these situations, but they show the existence of communication equilibria that lead to higher efficiency. Using a model of continuous contributions with two privately informed players, Agastya et al. (2007) show theoretically that individuals do not have an incentive to contribute to the public good without communication, but binary communication gives them incentives to provide the good with positive probability.

Kawamura (2011) uses an $n$-player setting and shows that there always exists an equilibrium where binary messages are credible and that, when $n$ goes to infinity, the equilibrium with binary communication is the most efficient one. Costa and Moreira (2012) find that a truthful equilibrium of the communication game with a binary message space cannot be Pareto dominated by any truthful equilibrium with any finite message space. They argue that this is because players use a threshold rule to make contribution decisions and in any situation with more than one contribution threshold, they have incentives to understate their types and thereby free-ride on their partners' investments.

\section{Experiments}

Threshold public goods games have been implemented in the laboratory by few researchers. Bagnoli and McKee (1991) find that the Pareto efficient outcome emerges when the cost of the good, the payoffs to those in the group, and the initial wealth positions of those in the group are common knowledge. They report that if the collective valuation of a group exceeds the cost of the good, the members of the group voluntarily contribute exactly the cost of the public good. Van de Kragt et al. (1983) show that free communication via general, unstructured discussion produces better outcomes than the same games conducted without communication in the context of public goods games similar to the one implemented by us. The message space was the entire English language and speaking order was entirely 
endogenous, occurring in continuous time with face-to-face communication. An important distinction from our game is that the contribution costs were equal for all players and common knowledge. Thus, Van de Kragt et al. (1983) eliminated two important impediments to coordination - private information and heterogeneity in costs. More structured cheap talk environments with public goods have been examined by Smith (1980) and Ferejohn et al. (1982). They considered the problem of designing market-like auction processes for the provision of a discrete public good.

Palfrey and Rosenthal (1991) report results from three-person threshold games and conclude that in the absence of communication, behavior is closely approximated by the Bayesian equilibrium predictions, except that subjects contribute slightly more often than predicted. In other experiments, they implemented a binary message stage prior to the decision making stage and found that this type of communication fails to provide more efficient outcomes than the 'no communication' outcome. They also found that subjects use a cutoff decision rule when it is optimal to do so in the 'no communication' treatment, while players' behavior is less systematic with communication.

Several experiments have implemented different forms of communication structures to evaluate the effect of cheap talk in other games. Cooper et al. $(1989,1992)$ studied one-way communication and two-way communication in coordination games and concluded that allowing pre-play communication does not uniformly lead to the play of the Pareto-dominant Nash equilibrium. There are situations where one-way communication performs significantly better than two-way communication. In a standard repeated $\mathrm{VCM}^{2}$ model, Bochet et al. (2006) vary the cheap talk message space and find that the treatment with exchange of numerical messages does not affect efficiency compared to the situation with no communication. However, both types of verbal communication, face-to-face and anonymous chat, increase cooperation ${ }^{3}$. Costa and Moreira (2012) implement a two-person contribution game and find evidence that larger finite message spaces do not provide efficiency gain relative to the binary communication structure.

\section{Theoretical Framework}

In this section, we use a mechanism design approach to develop theoretical bounds on the range of behavior and efficiency that can be achieved under the different communication regimes. There

\footnotetext{
${ }^{2}$ Under a voluntary contribution mechanism (VCM), individuals voluntarily allocate their initial holdings of resources into the production of public and private goods. Certain assumptions on the payoffs and utilities are made such that there exists a dominant strategy to not contribute anything to the production of the public good and "free-ride" on the contributions of others.

${ }^{3}$ The result that face-to-face communication increases contributions in repeated VCM experiments has been shown by various researchers, including Isaac and Walker (1988), Cason and Khan (1999), Brosig et al. (2003), Belianin and Novarese (2005). Ostrom and Walker (1991) also find the same result in repeated common property resource situations. More references are provided in a survey by Ledyard (1995).
} 
are four sources of inefficiency that can arise. The first is wasteful undercontribution, which arises when exactly one individual contributes and the public good is not provided. The second is wasteful overcontribution, which arises when all three individuals contribute. The public good is provided, but could have been provided at lower cost. Both of these inefficiencies are coordination failures, and can also arise where there is no private information and all players have the same cost to contribution. The third source of inefficiency is the classic free rider problem, whereby the public good is not provided even though, given the cost realizations, it would be efficient to provide it. The fourth source of inefficiency arises when the public good is provided with exactly two contributors, but their costs are not the two lowest. In this section we explore the extent to which communication can mitigate the efficiency losses from these four sources of inefficiency.

\section{The Environment}

A group consisting of $N$ persons is undertaking a project. Each group member is endowed with one indivisible unit of input, which may be either consumed or "contributed" to the production of the group project. The project succeeds if and only if at least $K$ units are contributed. The value of the project to any individual is normalized to equal 1 . The private value of the endowed unit of input to an individual $i$ is denoted by $c_{i}$. Each individual $i$ knows $c_{i}$ but only knows that the other players' $c$ 's are independent random draws from a cumulative distribution distribution $F$ on $[0, C]$, where $F$ is common knowledge and $C>0 . \quad F$ is admissible if $F(0)=0, F(C)=1$ and $F$ is continuously differentiable on $[0, C]$, and we assume throughout that $F$ is admissible. The utility for player $i$ with cost $c_{i}$ is given by:

$$
\begin{gathered}
1+c_{i} \text { if } i \text { does not contribute and at least } K \text { others contribute } \\
c_{i} \text { if } i \text { does not contribute and fewer than } K \text { others contribute } \\
1 \text { if } i \text { contributes and at least } K-1 \text { other contributes } \\
0 \text { if } i \text { contributes and and fewer than } K-1 \text { others contribute }
\end{gathered}
$$

Formally the four sources of inefficiency are the following: wasteful undercontribution, where exactly $k \in\{1, \ldots, K-1\}$ individuals contribute and the public good is not provided; excess contributions, where exactly $k \in\{K+1, \ldots, N\}$ contribute; underprovision, where the public good is not provided even though there exist $K$ individuals such that the sum of their costs is less than $N$ (the total social benefit of providing the public good); and cost inefficiency, where the public good is provided with exactly $K$ contributors, but their costs are not the $K$ lowest costs. Notice that underprovision can 
be particularly inefficient when at the same time there is also wasteful undercontribution. We next explore the extent to which a benevolent mechanism designer can mitigate the ex ante welfare losses from these four sources of inefficiency.

\section{Baseline Lower Bound: Equilibrium Without Communication}

We first consider the positive contribution symmetric Bayesian Nash equilibrium of the game without communication. ${ }^{4}$ This is a relevant lower bound for efficiency for all four treatments, and provides the upper bound as well for the no-communication treatment.

This Bayesian equilibrium is characterized by a cutpoint strategy, $c^{*}$ : player $i$ contributes if and only if $c_{i} \leq c^{*}$. In our case with a uniform distribution of contribution costs, $c^{*}$ solves the equation $c^{*}=\left[2 c^{*}\left(C-c^{*}\right] / C^{2}\right.$. The left hand side is the opportunity cost of contributing for a player a private cost of $c^{*}$ and the right hand side is the probability that the player's contribution is pivotal. In equilibrium these two are equal. Players with a cost below $c^{*}$ are better off contributing, given others are using the $c^{*}$ decision rule and players with private costs greater than $c^{*}$ are better off not contributing. Individuals with a cost of exactly $c^{*}$ are indifferent between contributing and not contributing. Thus, for $C=1$, the equilibrium is $c_{1.0}^{*}=.5$ and for $C=1.5$, the equilibrium is $c_{1.5}^{*}=.375$. We use these two values of $C$ in our experiment.

\section{Upper Bound: Optimal Communication Equilibrium}

With communication, many new additional equilibria arise. The most obvious class of equilibria are babbling equilibria, where the equilibrium strategies in the communication stage are such that no information is transmitted. In all of these equilibria, the behavior in the second stage is identical to the behavior in the game without communication, so these equilibria do not enhance efficiency. However, more interesting communication equilibria lead to higher ex ante payoffs for all players. Moreover, the set of communication equilibria of the game can depend on the message space and protocol of the communication stage. While there are multiple equilibria with communication ${ }^{5}$, in this section we identify efficiency bounds for each of the three communication protocols, and identify equilibrium strategy profiles that achieve these bounds. As introduced in section 1, the three communication protocols are binary communication, direct mechanism without a mediator through announcement of a number in the support of the distribution of contribution costs and natural language communication through text chat.

\footnotetext{
${ }^{4}$ There is also a zero contribution equilibrium that we ignore because it is unstable, as shown in Palfrey and Rosenthal (1991).

${ }^{5}$ Palfrey and Rosenthal (1991) characterize a class of equilibria in these games with binary communication.
} 
Equilibrium outcomes would be fully efficient if there were a communication equilibrium that always resulted in the individuals with the lower two costs contributing while the individual with the highest cost does not contribute. This 'first best outcome', however, is not consistent with equilibrium when $C=1$ as there would be incentives to over report costs. Furthermore, if $C=1.5$ then it is not consistent with equilibrium because ex post individual rationality would be violated: specifically, this 'first best outcome' could not be achieved when one or both of the lower two costs are greater than 1. Alternatively, when $C=1.5$ one might hope that a 'constrained first best outcome' would be supportable by a communication equilibrium, where (a) individuals with the lower two costs only contribute if both of the lower two costs are less than or equal to 1, and (b) no one contributes if one or both of the lower two costs are higher than 1. However, this would clearly violate incentive compatibility, for the same reasons the first best is not incentive compatible for $C=1$.

For the two communication mechanisms with essentially continuous message spaces (direct revelation and chat), we use mechanism design theory to characterize the optimal equilibrium. We model the communication game as direct mechanism in which we imagine that each individual independently submits a report of their private cost to the mechanism designer from the support of possible costs (either $[0,1]$ or $[0,1.5])$. The mechanism designer, after receiving all three reports, makes a recommendation to each player to either contribute or not. An incentive compatible and ex post individually rational mechanism is one in which, given the mechanism designer's recommendation strategy (which may be randomized), it is optimal for each player to honestly report their true private cost and to obey the recommendation of the mechanism designer. Among this class of mechanisms we identify the symmetric one that optimizes the ex ante group payoff. ${ }^{6}$ After this characterization, we turn to the binary communication games, where the characterization is different due to the restricted message space.

\section{The optimal mechanism design problem}

In this section we characterize the ex ante efficient equilibrium of the game with communication between the players by first characterizing an optimal mechanism. Consider a direct mechanism $(p, a)$ where $p:[0, C]^{N} \rightarrow[0,1]$ is the probability of provision as a function of the reported profile of values; and $\left\{a_{i}:[0, C]^{N} \rightarrow[0,1]\right\}_{i=1, \ldots, N}$ is the (possibly random) profile of contribution assignments. In the mechanism design problem, the optimal mechanism must satisfy a number of constraints. First, it must be incentive compatible, in the sense that it is a Bayesian equilibrium for all individuals to truthfully report their private information (cost of contribution). Second, feasibility requires that ( $p, a)$ satisfies

\footnotetext{
${ }^{6}$ Symmetry is without loss of generality.
} 
an expected budget constraint. That is, $(p, a)$ is feasible if and only if $p(\mathbf{c}) \leq \frac{1}{K} \sum_{i=1}^{N} a_{i}(\mathbf{c})$, for all profiles of individual costs, $\mathbf{c}=\left(c_{1}, \ldots, c_{N}\right)$. That is the expected sum of the payments must be at least $K$ times the probability the public good is produced. Third, in the actual contribution game that follows the communication stage, no individual can be forced to contribute if the payoff from contributing is negative. In particular, this requires at least that $a_{i}(\mathbf{c})=0$ for all $\mathbf{c} \in[0, C]^{N}$ such that $c_{i}>1$. We refer to this as the ex post individual rationality constraint. ${ }^{7}$ Without loss of generality we can restrict ourselves to symmetric mechanisms, so the $p$ functions and the $a$ functions are anonymous. We then work with the reduced form of the mechanism, denoted by $(P, A)$, where $P:[0, C] \rightarrow[0,1]$ is the expected probability of provision from an interim standpoint for an individual who has valuation $c_{i}$; and $A:[0, C] \rightarrow[0,1]$ is the expected probability of $i$ contributing from an interim standpoint for an individual who has valuation $c_{i}$. That is:

$$
\begin{aligned}
& P(c)=\int_{C_{-i}} p\left(c_{i}, c_{-i}\right) d F\left(c_{-i}\right) \\
& A(c)=\int_{C_{-i}} a\left(c_{i}, c_{-i}\right) d F\left(c_{-i}\right)
\end{aligned}
$$

Because feasibility implies that $p(\mathbf{c}) \leq \frac{1}{K} \sum_{i=1}^{N} a(\mathbf{c})$, for all $\mathbf{c} \in[0, C]^{N}$, the corresponding reduced form mechanism, $(P, A)$, also must satisfy the budget constraint in expectation, which can be written as: 8

$$
\frac{K}{N} \int_{0}^{C} P(c) f(c) d c \leq \int_{0}^{C} A(c) f(c) d c
$$

We refer to a mechanism as budget balanced if (1) holds with equality. We next proceed by characterizing the optimal reduced form mechanism, in two different cost distributions, which are used in the experiment.

\section{Low cost distribution: $C=1$}

The $C=1$ case is the simplest case $^{9}$ because in any budget balanced mechanism, the ex post individual rationality constraint will not be binding. We will derive the optimal mechanism below, without imposing the ex post individual rationality constraint, and then show that the optimal mechanism is

\footnotetext{
${ }^{7}$ This is only a necessary condition for ex post individual rationality, but it is the only one that is relevant for our characterization.

${ }^{8} \mathrm{In}$ principle, there is also the question about whether additional implementability conditions may impose further constraints on the problem, i.e., the question of whether the optimal reduced form mechanism can be feasibly implemented by a $(p, a)$ mechanism. As it turns out, for this class of problems it is not an issue.

${ }^{9}$ The results for this case extend easily to any $C$ such that $0<C \leq 1$, as shown in the appendix.
} 
budget balanced and hence the ex post individual rationality constraint is not binding. Thus, for the case of $C=1$ the optimal mechanism is characterized as the solution to the following program:

$$
\begin{aligned}
& \max _{(P, A)} \int_{0}^{1} U(c) d c \\
& \text { subject to } \\
& U(c) \geq \\
& P\left(c^{\prime}\right)-c A\left(c^{\prime}\right) \forall c, c^{\prime} \\
& \frac{K}{N} \int_{0}^{1} P(c) d c \leq \int_{0}^{1} A(c) d c \\
& 0 \leq P(c) \leq 1 \forall c, 0 \leq A(c) \leq 1 \forall c
\end{aligned}
$$

where $U(c) \equiv P(c)-c A(c)$ is the interim expected utility to an individual with private cost $c$.

The first constraint is incentive compatibility and the other constraints are feasibility. Following standard arguments from Bayesian mechanism design (see for example Ledyard and Palfrey 2002), one can show that the solution to this optimization problem is the full provision lottery draft mechanism. That is, the public good is always produced and a random subset of $K$ individuals contribute, without regard to individual cost realizations. Thus, the two coordination problems (undercontribution and overcontribution) and the underprovision inefficiency are perfectly solved, but the fourth source of inefficiency, cost inefficiency, is ignored. The reason cost inefficiency is ignored is that the only way to sort out lower cost types from higher cost types is to sometimes fail to produce the public good. That is solving the cost inefficiency problem would require reducing the probability the public good is provided. But the cost of failing to produce the public good is extremely high. In particular, it is very high relative to the benefits of shifting the cost burden in the direction of lower cost agents. This result is summarized in the following proposition.

Proposition 1. If costs are uniformly distributed on $[0,1]$, then the solution to (2) is $P(c)=1$ and $A(c)=\frac{K}{N}$ for all $c \in[0,1]$.

Proof. See Appendix A, which proves a more general result for which this is a special case.

\section{High cost distribution: $C=1.5$}

If the cost distribution admits $c_{i}>1$, as is the case when $C=1.5$, then ex post individual rationality constraints play a role in characterizing the optimal communication mechanism, because it is irrational for any individual with a $\operatorname{cost} c_{i}>1$ to contribute, since the individual benefit is only 1 . Thus, the solution of (2) characterized in Proposition 1 violates ex post individual rationality when $C>1$, and the analysis becomes more complicated. 
We can write the ex post individual rationality constraint in the reduced form as:

$$
A(c)=0 \forall c>1
$$

This constraint also implies that $P(c)$ must be constant for all $c>1$, and we will denote this expected provision for high types as $\underline{P}$. Denote by $c^{*}=\inf \{c \mid A(c)=0\} \leq 1$ and observe that $U(c)=\underline{P}$ $\forall c \in\left[c^{*}, 1.5\right]$. We know $c^{*}$ exists, because $A(c)=0 \quad \forall c>1$ and $A^{\prime}(c) \leq 0$. Denote the portion of $P$ and $A$ defined on $c \leq c^{*}$ by the functions $\widehat{P}:\left[0, c^{*}\right] \rightarrow[0,1]$ and $\widehat{A}:\left[0, c^{*}\right] \rightarrow[0,1]$. With this notation, we write the optimization problem as:

$$
\begin{aligned}
& \max _{\left(\widehat{P}, \widehat{A}, \underline{\left.\underline{P}, c^{*}\right)}\right.} \int_{0}^{1.5} U(c) \frac{1}{1.5} d c \\
& \text { subject to } \\
U(c) & =\underline{P} \forall c \in\left[c^{*}, 1.5\right] \\
\widehat{A}(c) & =-U^{\prime}(c) \forall c \in\left[0, c^{*}\right] \\
U^{\prime \prime}(c) & \geq 0 \forall c \in\left[0, c^{*}\right] \\
\frac{K}{N}\left\{\underline{P}\left[1-F\left(c^{*}\right)\right]+\int_{0}^{c^{*}} \widehat{P}(c) \frac{1}{1.5} d c\right\} & \leq \int_{0}^{c^{*}} \widehat{A}(c) \frac{1}{1.5} d c \\
0 & \leq \widehat{P}(c) \leq \underline{P} \forall c, 0 \leq \widehat{A}(c) \leq 1 \forall c \in\left[0, c^{*}\right]
\end{aligned}
$$

Following arguments similar to the proof of Proposition 1, we can characterize the optimal communication mechanism as a solution to (3). The mechanism is not perfectly flat, as was the case for $C=1$, but it is as flat as possible, given the binding constraint imposed by ex post individual rationality. Specifically, $P$ and $A$ have two flat components, which are separated at a critical cost level, $c^{*}$. Individuals with cost above $c^{*}$ never contribute. Individuals with cost below $c^{*}$ all contribute with the same probability.

Proposition 2. If costs are uniformly distributed on $[0, C]$ and $C=1.5, K=2, N=3$ then the solution to (2) is given by:

$$
\begin{aligned}
c^{*} & =0.75 \\
P(c) & =0.75 \forall c \in\left[0, c^{*}\right] \\
& =0.25 \forall c \in\left[c^{*}, 1.5\right] \\
A(c) & =\frac{2}{3} \forall c \in\left[0, c^{*}\right] \\
& =0 \forall c \in\left[c^{*}, 1.5\right]
\end{aligned}
$$




\section{Proof. See Appendix A.}

This mechanism solves the first two problems of miscoordination, because budget balance is always satisfied. On the other hand, the existence of an ex post individual rationality constraint means that the public good is not always provided, so the free rider problem is not fully solved. In fact, because $C=1.5$ and only two contributors are required, it is always efficient to provide the public good because the total cost of providing it is always less than 3, which is equal to the sum of public good benefits to the group. The cost inefficiency problem is also not solved in this mechanism. As with the earlier case where $C \leq 1$, solving the cost inefficiency problem would require reducing the probability the public good is provided. The cost of reducing the provision probability is very high relative to the benefits of shifting the cost burden to the lower cost agents.

Binary Communication Cutpoint Equilibrium (CCE) The optimal mechanisms described above cannot be implemented with a single round of simultaneous binary communication, which is the simplest possible message space we explore in the experiment. The message space is just not rich enough to implement a jointly controlled lottery to randomly selecting exactly $K$ contributors when more than $K$ individuals indicate a willingness to contribute. An earlier paper (Palfrey and Rosenthal (1991)) identifies a class of cheap talk equilibrium when $\mathrm{K}=2$ and $\mathrm{N}=3$, which we call binary communication cutpoint equilibrium. It remains an unproven conjecture that these equilibria are the most efficient symmetric equilibria of the communication game with one round of simultaneous binary communication. The message space is $\{0,1\}$ and the equilibrium is characterized by two cutpoints, $c_{c}$ and $c_{3}$. In the first stage, player $i$ reports " 1 " if and only if $c_{i} \leq c_{c} \cdot{ }^{10}$ Behavior in the second stage depends on how many players reported " 1 " in the first stage. If 0 or 1 players reported "1" then nobody contributes in the second stage. If exactly 2 players reported " 1 " in the first stage, then those two players contribute in the second stage and the third player does not contribute. If all 3 players reported " 1 " in the first stage, then those players use the cutpoint $c_{3}<c_{c}$ as their strategy in the second stage: i.e., contribute if and only if $c_{i} \leq c_{3} .{ }^{11}$

The equilibrium is characterized by two equations, one for each cutpoint. The condition in the cheap talk stage is that a player with $c_{i}=c_{c}$ is indifferent between reporting " 1 " and " 0 ", given the equilibrium continuation in the second period. If all other players use $\left(c_{c}, c_{3}\right)$ the expected payoff of

\footnotetext{
${ }^{10}$ Thus, reporting a "1" is interpreted in equilibrium as a conditional promise to contribute.

${ }^{11}$ Obviously such equilibria can also be implemented with any richer message space, including our direct revelation message space and the text chat message space.
} 
announcing "0" for a player with $c_{i}=c_{c}$ equals:

$$
\left(\frac{c_{c}}{C}\right)^{2}
$$

and the expected payoff for announcing " 1 " is:

$$
\left(\frac{c_{c}}{C}\right)^{2}\left(\frac{c_{3}}{c_{c}}\right)^{2}+2\left(\frac{c_{c}}{C}\right)\left(1-\frac{c_{c}}{C}\right)\left(1-c_{c}\right)
$$

Equating these expressions and collecting terms gives:

$$
\left(c_{c}\right)^{2}=\left(c_{3}\right)^{2}+2\left(c_{c}\right)\left(C-c_{c}\right)\left(1-c_{c}\right)
$$

The cutpoint in the contribution stage, $c_{3}$, is characterized by the player with $c_{i}=c_{3}$ being indifferent between contributing and not contributing, given everyone else is using equilibrium strategies. This indifference equation is given by:

$$
2\left(\frac{c_{3}}{c_{c}}\right)\left(1-\frac{c_{3}}{c_{c}}\right)=c_{3}
$$

which reduces to:

$$
c_{3}=c_{c}-\frac{c_{c}^{2}}{2}
$$

The equilibrium is obtained by simultaneously solving (7) and (8) for $\left(c_{c}, c_{3}\right)$. For our parameters $(C=1$ and $C=1.5)$, the binary communication equilibrium cutpoints are summarized in Table 1.

\begin{tabular}{l|cr}
$C$ & $c_{c}$ & $c_{3}$ \\
\hline 1 & 0.608 & 0.426 \\
1.5 & 0.724 & 0.462 \\
\hline
\end{tabular}

Table 1: Binary communication equilibrium cutpoints.

It is interesting to note that the CCE does not eliminate all the miscoordination inefficiencies. It is possible for exactly one player to contribute, if his value is less than $c_{3}$ and the other two players have values between $c_{3}$ and $c_{c}$. Given the parameters of the experiment the chance this happens in the CCE is very low: probability 0.05 for $C=1$ and probability 0.03 for $C=1.5$. Similarly, it is possible for overcontribution in equilibrium, if all three players have values less than $c_{3}$. Given the parameters of the experiment the chance this happens in the CCE is also very low: probability 0.07 
for $C=1$ and probability 0.03 for $C=1.5$. Hence the welfare losses from such miscoordination is very low in expected terms, partly because the event is unlikely and partly because the value of the wasted contribution is low.

Compared to the optimal mechanism with unlimited communication, the welfare losses in the communication equilibrium are still very large because of the high probability of non-provision. The probability of non-provision is approximately 0.39 for $C=1$, which is nearly as great as the probability of non-provision in the symmetric Bayes Nash equilibrium (0.50), and far worse than the optimal mechanism, which always produces the public good. For $C=1.5$ the probability of non-provision is 0.56, which is much less than the probability of non-provision in the symmetric Bayes Nash equilibrium, which is 0.84 . Thus the potential gains from binary communication are greater for $C=1.5$ than for $C=1$.

\section{Experimental Design and Hypotheses}

The experimental design, procedures and treatments are discussed in Section 4.1. Section 4.2 lists the hypotheses that are formally tested.

\subsection{Design, Procedures and Treatments}

The experiments were conducted at the California Social Science Experimental Laboratory (CASSEL), University of California, Los Angeles (UCLA) using the Multistage software package. Participants were recruited from a pool of volunteer subjects, maintained by CASSEL. Upon arrival, instructions were read aloud. Subjects interacted anonymously with each other through computer terminals. Sessions lasted from 30 to 50 minutes and participants earned on average US $\$ 18.47$ in addition to a show-up fee of $\mathrm{US} \$ 5^{12}$.

In all the experiments, we set $K=2$ and $N=3$. Each individual $c_{i}$ is an independent random draw from a uniform distribution on $[0, C]$, and the experimental design allows for two values of $C$ (1 and 1.5) corresponding to the two cases analyzed in the theoretical section of the paper. $C=1$ means that it is common knowledge that everyone has costs lower than the public benefit and hence, no one has a dominated contribution strategy. However, in the situation with $C=1.5$, individuals have a strictly dominant strategy not to contribute whenever $c_{i}>1$, implying the problem of ex post individual rationality in the communication equilibria.

The communication structure was varied in each of the two parametric configurations, $C=1$ and $C=1.5$. We used four communication treatments: "no communication", "binary communication",

\footnotetext{
${ }^{12}$ Payoffs ranged from US $\$ 11$ to US $\$ 25.50$ with a standard deviation of US $\$ 3.18$.
} 
"direct revelation", and "unrestricted text chat", resulting in a $2 \times 4$ design. We ran two sessions for each of these eight treatments, thus a total of 16 sessions, with 9 to 15 subjects per session. There were 24 subjects per treatment, except for $C=1$ unrestricted text chat (18 subjects) and $C=1$ no communication (21 subjects), for a total of 183 subjects. No subject participated in more than one session. The details of the experimental protocols are briefly discussed below ${ }^{13}$. Each session consisted of 20 rounds that followed an initial unpaid walk-through practice round to explain the computer interface. After a round was over, participants were randomly rematched into new three person groups and everyone was independently and randomly assigned new costs. The random rematching was done to limit the reputation and super-game effects which might occur with repeated play.

\section{No Communication}

Contribution costs were implemented as opportunity costs. In each round, each subject was allocated a single indivisible "token", which had a private value that was referred to as a "token value". Subjects were informed that token values in integer increments between 1 to $100 C$ points are independently drawn with replacement from identical uniform distributions and randomly assigned to subjects. Each subject was informed of her token value but knew only the probability distribution of other subjects' token values. Each subject was then asked to enter a decision to spend or keep their token. If at least two of the three subjects spent their token, then each subject received 100 points if she had chosen to spend her token, while the payoff was 100 points plus her token value if she had chosen to keep her token. Subjects earned US $\$ 1$ for every 100 points they accumulated during the experiment. If a subject chose to spend but none of her other group members spent their tokens then that subject earned 0 points in the round. If a subject chose not to spend and fewer than two other group members spent their tokens then that subject earned her token value in the round. ${ }^{14}$

\section{Binary Communication ("Binary")}

Each round had two stages: a communication stage and a contribution stage. In the communication stage, subjects chose one of the two messages: "I intend to spend my token"; "I intend to keep my token". They were advised that these messages were not binding, and they could make either contribution decision regardless of which message they sent in the communication round. After these binary messages were sent, each person was told how many members in their group sent each message,

\footnotetext{
${ }^{13}$ For details, please see a sample copy of the instructions provided in Appendix B.

${ }^{14}$ Due to an error in the computer program, no subject received a token value equal to 100 in the $C=1$ treatment and 150 in the $C=1.5$ treatment; the actual distribution was uniform from 1 to 99 or 149, respectively.
} 
and was reminded which message he or she had sent. This was directly followed by the contribution stage, where individuals made binding contribution decisions.

\section{Direct Revelation ("Token")}

Each round again had a communication stage and a contribution stage. In the communication stage, subjects had 20 seconds to send a message to the other members of her group. They were told that this message can only be an integer between 1 and $100 C$ and each member was allowed to send only one such message. Thus, the message space in this treatment corresponds to the direct mechanism and is much larger than the "binary communication" sessions. Each subject observed the messages sent by her group members. Each subject was also told that in the event she did not send any message, the other members of her group would see a "? " against her subject id at the end of the 20 seconds. After the communication stage was over, individuals made contribution decisions. We called this treatment "token" because subjects had the opportunity to reveal their true token values. If token values were revealed truthfully, there would be the opportunity for perfect coordination in the $C=1$ condition, with the two individuals with the lowest token values contributing. In principle, the same could occur in the $C=1.5$ condition, provided at least two individuals had token values no greater than 100 .

\section{Unrestricted Broadcast Text Chat ("Chat")}

This treatment also consisted of 20 rounds and each round had two stages, with the only difference being in the structure of the communication stage. Prior to the contribution stage, every group had a discussion period which lasted 60 seconds, during which subjects could send messages to the other members of the group. Individuals were told that the messages had to conform to certain rules, including that they must be relevant to the experiment and subjects should not send messages intended to reveal their identity. Thus, the message space under this treatment is 'anonymous, unrestricted and unstructured text messages' and is much larger than the finite message space under the "token revelation" sessions. Importantly, this treatment gave subjects the opportunity to employ natural language.

\subsection{Hypotheses}

We test several hypotheses regarding (a) the effect of communication on efficiency, (b) comparison across communication treatments differing in the richness of message space, and (c) differences across $C=1$ and $C=1.5$ sessions.

These hypotheses are based on comparing the best communication equilibrium achievable under 
the different communication schemes, as derived in Section 3. For easy reference Table 2 below summarizes the theoretical results about the four different kinds of inefficiencies for our experimental parameters, under the different treatments. Note that the least efficient communication schemes (no communication and binary) perfectly solve the cost inefficiency problem, in contrast to the lottery draft mechanism. This reflects the fact that the cost inefficiency problem is second order in welfare loss compared to underprovision.

\begin{tabular}{|c|c|c|c|c|c|}
\hline $\begin{array}{c}\text { Inefficiency } \\
\text { Type }\end{array}$ & $\begin{array}{l}\text { First } \\
\text { Best }\end{array}$ & $\begin{array}{l}\text { No Comm. } \\
\text { (Bayes-Nash) }\end{array}$ & $\begin{array}{c}\text { Binary } \\
\text { (Binary Cutpoints) }\end{array}$ & Token & Chat \\
\hline$\underline{C=1}$ & & $c^{*}=0.50$ & $c_{c}^{*}=0.608 c_{3}^{*}=0.426$ & Lottery Draft & Lottery Draft \\
\hline Undercontribution & 0 & 0.375 & 0.05 & 0 & 0 \\
\hline Overcontribution & 0 & 0.125 & 0.07 & 0 & 0 \\
\hline Underprovision & 0 & 0.50 & 0.39 & 0 & 0 \\
\hline Cost inefficiency & 0 & 0 & 0 & 0.67 & 0.67 \\
\hline$\underline{C=1.5}$ & & $c^{*}=0.375$ & $c_{c}^{*}=0.724 c_{3}^{*}=0.462$ & & \\
\hline Undercontribution & 0 & 0.42 & 0.03 & 0 & 0 \\
\hline Overcontribution & 0 & 0.02 & 0.03 & 0 & 0 \\
\hline Underprovision & 0 & 0.84 & 0.56 & 0.50 & 0.50 \\
\hline Cost inefficiency & 0 & 0 & 0 & 0.17 & 0.17 \\
\hline
\end{tabular}

Table 2: Theoretical ex-ante probabilities of different forms of inefficiency for $C=1$ and $C=1.5$.

The effect of communication on efficiency is broken down into four separate hypotheses with respect to the total earnings generated, the likelihood of public good provision, the number of contributors and the costs of contributors. First, as there exist equilibria under communication that have higher payoffs for players than in the Bayesian Nash equilibrium without communication, one can conjecture that the earnings would indeed be higher if communication is allowed. For similar reasons, we expect the public good to be provided more often if there is pre-play communication than without any communication communication. These give rise to our first two hypotheses:

Hypothesis 1 (Earnings Hypothesis). Total earnings are higher with communication than without communication.

Hypothesis 2 (Provision Hypothesis). The likelihood of public good provision is greater in the communication sessions than in the non-communication sessions.

The ex ante efficient solution is for just two individuals to contribute their token, and for the con- 
tributors to have the two lowest costs while the free rider has the highest cost (ignoring ties). Without communication, it is impossible for players to know who has relatively high valuations and who has relatively low ones. Thus the "efficient" outcome can occur only by chance. However, with sufficient communication, it is at least feasible to coordinate decisions in a way that produces this desired outcome. Thus, we can expect to have fewer wasteful contributions, that is, fewer incidences of one or three individuals contributing in the case with communication. In other words, communication leads to lower production inefficiency by lowering both over and under-contributions. However, the fourth form of inefficiency, i.e., cost inefficiency is higher with communication than under no communication (see Table 2). Hence, we have the following hypotheses:

Hypothesis $3 a$ (Under-contribution Hypothesis). The incidence of exactly one person in a group contributing is lower in the communication sessions than in the non-communication sessions.

Hypothesis $3 b$ (Over-contribution Hypothesis). The incidence of all members in a group contributing is lower in the communication sessions than in the non-communication sessions.

Hypothesis 4 (Cost [In]efficiency Hypothesis). Conditional on exactly two contributing, the incidence of contributors not being the members with the lower two costs is higher with communication than under no communication.

We study three different forms of communication. While the binary sessions use an exchange of binary messages, the token sessions have a much larger although finite message space, including a null message. The 'text chat', while anonymous, uses natural language in continuous time. Hence, we have a progression in the treatments in terms of the richness of the message space implemented. One would expect that it would be easier to implement efficient outcomes as the message space becomes richer and this leads to a hypothesis that there will be a monotonic relation between efficiency and richness of the message space: ${ }^{15}$

Hypothesis 5 (Monotone Hypothesis). Efficiency increases with the richness of the message space. It is highest in the chat treatment, followed by the token treatment, followed by the binary treatment, and lowest in the no communication treatment.

The final hypothesis compares the provision of public good across the two different $C$ sessions. When it is common knowledge that every member's cost is less than the benefit from the public good, $100 \%$ provision of public good is possible. However, in the $C=1.5$ case, it is no longer possible to provide the public good all the time assuming subjects do not use dominated strategies. Indeed, when at least two of the subjects have costs greater than 100, provision would violate individual rationality. This event occurs with probability $7 / 27$. This leads to the following hypothesis:

Hypothesis 6 (C Hypothesis). For each communication protocol, the probability of public good

\footnotetext{
${ }^{15}$ This hypothesis, while intuitive, does not follow directly from the theory, in contrast to the other five hypotheses.
} 
provision is greater in the $C=1$ sessions than in the $C=1.5$ sessions.

\section{$5 \quad$ Results}

Here we first present the results from testing the specific hypotheses that we discussed earlier. Subsection 5.2 compares the performance of the data in all treatments to the various theoretical benchmarks. The final subsection briefly discusses the behavior in the communication stages of the different treatments.

\subsection{Group Outcomes and Test of Hypotheses}

The first hypothesis involves a comparison between the average earnings without communication and the average earnings with the three communication treatments. Table 3 documents the average group earnings net of token values in rounds 11-20 for the four treatments, in both $C$ sessions. We use the net earnings as opposed to gross earnings to reduce the 'randomness' present due to the realization of the drawn token values. ${ }^{16}$ In the $C=1$ sessions, average net earnings are higher with communication in both the 'token' and the 'chat' treatments, but the difference is significant only for the 'chat' treatment. The other two communication treatments yield similar earnings as those under 'no communication'. In the $C=1.5$ sessions, 'chat' results in higher earnings only in the second half of the experiment.

\begin{tabular}{ccccc}
\hline \hline & No Comm. & Binary Comm. & Token Revelation & Chat \\
\hline Provision rate & 0.43 & $\frac{C=1}{0.41}$ & 0.50 & $0.98^{* * * *}$ \\
Group earnings & 88.0 & 80.2 & 101.5 & $221.1^{* * *}$ \\
Observations & 70 & 80 & 80 & 60 \\
& & $\underline{C=1.5}$ & & \\
Provision rate & 0.28 & 0.38 & 0.25 & $0.43^{* *}$ \\
Group Earnings & 39.6 & 58.7 & 42.1 & $83.4^{* * *}$ \\
Observations & 80 & 80 & 80 & 80 \\
\hline
\end{tabular}

* significant at $10 \%$;* significant at $5 \% ; * * *$ significant at $1 \%$.

Table 3: Average group earnings net of token values and frequency of public good provision. Significance levels are for differences between the communication treatment and 'No Communication'.

Taking each group as an independent observation, one-tailed Mann-Whitney tests show that there is no significant difference in the net group earnings between 'binary communication' and 'no commu-

\footnotetext{
${ }^{16}$ Token values are also netted out because the exact draw of token values varies from treatment to treatment. Thus our measure of group earnings in this table is relative to a benchmark of 0 contributions. Unless otherwise noted, throughout the results section, we consider only the data from rounds 11-20 for performing the statistical analyses. Tables reporting the results for the first 10 round and for all 20 rounds combined are in Appendix C.
} 
nication' treatments and between 'token revelation' and 'no communication' treatments. ${ }^{17}$ However, the differences are statistically significant at the $1 \%$ level only between 'no communication' and 'chat' implying that subjects end up with higher earnings only when they are provided with unstructured communication in the form of text chat. This significant difference is true for both $C$ sessions. ${ }^{18}$ The above discussion leads us to support Earnings Hypothesis and conclude the following:

Result 1. Total earnings are significantly higher with communication only in the chat condition (H1).

Table 3 also reports frequencies of the public good provision for rounds 11-20 in each of the four treatments. An immediate finding is that in line with the previous result, not all forms of communication increase the likelihood of the good being provided. Clearly, 'binary communication' and 'token revelation' sessions result in either similar or lower public good provision when compared to 'no communication' sessions, considering the two $C$ sessions. It is only in the 'chat' treatment that the provision of good is higher in both $C=1$ and $C=1.5$ sessions. A difference in proportions z-test for each of the binary comparisons of the 'communication' sessions with the 'no communication' sessions shows that there is significant difference between 'unrestricted chat' and 'no communication' in both $C$ sessions (at $5 \%$ level ${ }^{19}$. Supporting the Provision Hypothesis, we have the following:

Result 2. The likelihood of public good provision is significantly greater with communication only in the chat condition, for both cost treatments (H2).

Next, we turn to the following question: Does communication help in reducing production inefficiency in the form of wasteful contributions? While the frequency with which exactly one group member contributes is always lower with communication, the difference is significant only for the 'chat' treatment (see Table 4). With respect to overcontribution where all three individuals in a group contribute, communication helps in all forms of communications but only for $C=1$ sessions. So, communication helps in reducing production inefficiency through both the lowering of undercontribution as well as overcontribution. The percentage of over-contribution is already low without any communication. Thus, supporting Under-contribution Hypothesis and Over-contribution Hypothesis, we have the following results:

\footnotetext{
${ }^{17}$ The statistical tests treat group observations as independent because there are too few sessions to cluster by sessions. To minimize the possibility of correlation due to learning over time through shared histories over the 20 rounds of a session, the random matching into groups across periods is anonymous and there are at least three parallel groups in every session. In any case, very little learning in the aggregate is observed over time in these experiments, as behavior in the first half of sessions is generally similar to behavior in the second half of the experiment with no significant improvement across time except in the $C=1.5$ chat treatment, where the improvement is small in magnitude. See the additional tables in the Appendix that compare the first 10 and last 10 rounds of data for all the treatments.

${ }^{18} \mathrm{In}$ fact, the behavior in the two sessions is nearly the same. Both have provision rates approaching $100 \%$.

${ }^{19}$ These are results from one-tailed tests of difference in two independent proportions. For the $C=1$ situation, the data numbers do not satisfy the criteria: $n(1-p)>5$, where $n$ is the number of observations and $p$ is the proportion. But its easy to infer that the proportion is almost 1 under 'unrestricted chat' and hence there would be a significant difference when compared with the 'no communication' session.
} 
Result 3a. For both cost treatments, the incidence of exactly one person in a group contributing is lower in all communication treatments. It is significantly lower in the chat condition (support for Н3а).

Result 3b. For both cost treatments, the incidence of all three persons in a group contributing is lower in all communication treatments. It is significantly lower only in the $C=1$ chat condition (support for H3b).

\begin{tabular}{ccccc}
\hline \hline Contributors & No Comm. & Binary Comm. & Token Revelation & Chat \\
\hline & & $\frac{C=1}{17.4}$ & 9.9 & $0.0^{* * *}$ \\
0 & 11.4 & 41.3 & 40.0 & $1.7^{* * *}$ \\
1 & 45.7 & 35.0 & 38.8 & $96.6^{* * *}$ \\
2 & 28.6 & $6.3^{*}$ & $11.3^{*}$ & $1.7^{* * *}$ \\
3 & 14.3 & 80 & 80 & 60 \\
nobs & 70 & $\underline{C=1.5}$ & & \\
& & 23.7 & 35.0 & 27.5 \\
0 & 24.9 & 38.8 & 40.0 & $30.0^{* *}$ \\
1 & 47.5 & 35.0 & 17.5 & 37.5 \\
2 & 20.0 & 2.5 & 7.5 & 5.0 \\
3 & 7.6 & 80 & 80 & 80 \\
nobs & 80 & & & \\
\hline
\end{tabular}

* significant at $10 \% ; * *$ significant at $5 \%$; ** significant at $1 \%$. These significance levels are for difference in proportions z-tests across the communication treatment and 'No Communication'.

Table 4: Frequency distribution of number of contributors in percentages, communication treatment.

Results $3 \mathrm{a}$ and $3 \mathrm{~b}$ clearly illustrate one of the most fundamental roles of communication in these threshold public goods games: communication improves coordination. When either two or zero members of the group contribute, there is no miscoordination. There is free riding in the case of zero contribution, but no wasted contributions. With exactly two contributors, there may be a small inefficiency due to the private information about costs if the two contributors do not have the two lowest costs, but there is no miscoordination and no wasted contributions. In contrast, when one or three members of the group contribute, inefficiencies result purely from miscoordination, resulting in deadweight loss. These miscoordination outcomes correspond to the first two kinds of inefficiency identified in the theoretical section (undercontribution and overcontribution).

Finally, to combine results $3 \mathrm{a}$ and $3 \mathrm{~b}$, we compare the frequency of coordinated outcomes $(0$ or 2 contributors) and the frequency of coordination failures ( 1 or 3 contributors) across the four communication treatments in the two cost treatments. This comparison is shown in Table 5. Communication clearly leads to meaningful increases in coordination in all cases. The percentage increases in coordinated outcomes relative to no communication range from a $17 \%$ increase in the case of 'token 
revelation' with $C=1.5$ to a $141 \%$ increase in the case of 'chat' with $C=1$. Five out of six increases relative to no communication are statistically significant.

\begin{tabular}{ccccc}
\hline \hline $\mathrm{C}$ & No Comm. & Binary Comm. & Token Revelation & Chat \\
\hline $\mathrm{C}=1$ & 40.0 & $52.4^{* * *}$ & $48.7^{* *}$ & $96.6^{* * *}$ \\
$\mathrm{C}=1.5$ & 44.9 & $58.7^{* * *}$ & 52.5 & $65.0^{* * *}$ \\
\hline
\end{tabular}

* significant at $10 \% ;{ }^{* *}$ significant at $5 \% ; * * *$ significant at $1 \%$. These significance levels are for difference in proportions z-tests across the communication treatment and 'No Communication'.

Table 5: Frequency of coordinated outcomes (0 or 2 contributors).

The most efficient outcome occurs when exactly two people in a group contribute and those two have the lower two costs. As explained earlier, we define cost inefficiency as arising when those two contributors are not the two lowest cost members of the group. Table 6 documents the percentage of times the contributors are not the members with the lower two costs across the four treatments and for each $C$ session, conditional on exactly two contributors. The numbers are quite low for all treatments and there is no significant effect of communication, contrary to Hypothesis 4. This is particularly interesting for the chat communication treatment with $C=1$. In theory, with the optimal lottery draft, there would be a $2 / 3$ chance that the two contributors are not the members with lowest two costs, but we observe this happening between $10 \%$ and $20 \%$ of the time. The chat communication in the groups partially solved the cost inefficiency problem, as well as the underprovision problem.

Result 4. Cost inefficiency is not significantly higher in the communication treatments compared to no-communication (reject $\mathrm{H}_{4}$ ).

\begin{tabular}{ccccc}
\hline \hline $\mathrm{C}$ & No Comm. & Binary Comm. & Token Revelation & Unrestricted Chat \\
\hline$\underline{C=1.0}$ & $15.0(20)$ & $32.1^{*}(28)$ & $16.1(31)$ & $12.1(58)$ \\
$\underline{C=1.5}$ & $6.3(16)$ & $25.0^{*}(28)$ & $21.4(14)$ & $10.0(30)$ \\
\hline
\end{tabular}

* significant at $10 \%$;* significant at $5 \%$; ** significant at $1 \%$. These significance levels are for difference in proportions z-tests across the communication treatment and 'No Communication'.

Table 6: Percentage of times the contributors are not the members with the lower two costs, conditional on exactly two contributing. Number of observations where exactly two contribute in parentheses.

Summarizing the above results, we find that the structure of communication is crucial in determining whether there are significant gains over the situation where communication is not possible. The simultaneous exchange of binary messages aimed at disclosing intentions as well as the one-time broadcasting of a numerical message lead to some improvements, particularly with respect to a reduction in the frequency of miscoordination, but these gains are for the most part not large enough 
in magnitude to significantly improve average earnings and the probability of public good provision. With these coarser communication structures, groups also are generally not successful in bringing the members with the lowest two token values to contribute. Only when a rich form of continuous time communication with a "common language" is possible, are there significant gains in efficiency. While these gains are enormous (approaching the first best outcomes) in the $C=1$ treatment where it is common knowledge that everyone has a cost that is less than the benefit from the public good, these gains are harder to achieve in the $C=1.5$ treatment, where some individuals can have private costs that exceed the benefit.

Given results 1-4, it is now straightforward to test Hypothesis 5. Whether it is measured with respect to the average group earnings, likelihood of public good provision, incidence of wasteful contributions, or the subjects with lower two costs contributing, there is no strict progression in efficiency as we move from no communication to binary to a larger numerical message space to finally the "infinite" communication structure. The relation between efficiency and the richness of message space is weakly monotonic, but not strictly monotonic as hypothesized.

Result 5. Efficiency does not increase monotonically in the richness of the message space of the communication stage (reject H5). It is significantly higher only for the chat treatment.

The final hypothesis compares the likelihood of providing the public good in $C=1$ and $C=1.5$ sessions. There were 489 instances out of 1918 total observations (that is $25.5 \%$ of the cases) when at least two of the subjects in a group had costs higher than 100 in the $C=1.5$ sessions. The public good was provided in 6 out of 114 observations in the 'binary communication' treatment. In the other treatments, the good was never provided when there was only one person in a group having a cost less than 100 (out of a total of 375 such instances). These numbers strongly support Hypothesis 6 . As was shown in Table 3, public good provision is much higher in the $C=1$ sessions than in the corresponding $C=1.5$ sessions. The differences are significant at the $1 \%$ level for 'direct revelation' communication and 'chat', and at the $5 \%$ level for the 'no communication' sessions.

Result 6. Keeping the communication protocol fixed, the probability of public good provision is greater in the $C=1$ sessions than in the $C=1.5$ sessions (support for $\mathrm{H} 6$ ).

\subsection{The Quantitative Effects of Communication on Normalized Efficiency}

We now turn to the analysis of how the outcomes compare with the theoretical bounds discussed in Section 3 as well as the first-best outcome. Figure 1 displays normalized efficiency, defined as group earnings net of token values as a percentage of first-best group earnings net of token values. The graph displays the data (with 95 percent confidence intervals) and also the normalized efficiency 
in the optimal communication mechanisms (lottery drafts) characterized in Section 3, based on the actual cost draws in the data. The normalized efficiency of the Bayesian Nash equilibrium for each treatment are marked on the graph with horizontal lines, also based on the actual cost draws. The left panel is for the $C=1$ data and the right panel is for the $C=1.5$ data.

The normalized efficiency results can be summarized as follows. First, in all cases, groups do much better than if nobody contributed. That is, normalized efficiency is significantly greater than zero in all eight treatments. Second, with the exception of the chat communication treatments ${ }^{20}$, normalized efficiency is significantly lower than in the optimal mechanism. Third, in all of the $C=1$ treatments except with chat communication, efficiency is lower than in the Bayes-Nash equilibrium. Fourth, in contrast to the third finding, for the $C=1.5$ data, normalized efficiencies are always higher than the Bayes Nash equilibrium in every communication treatment (including no communication).
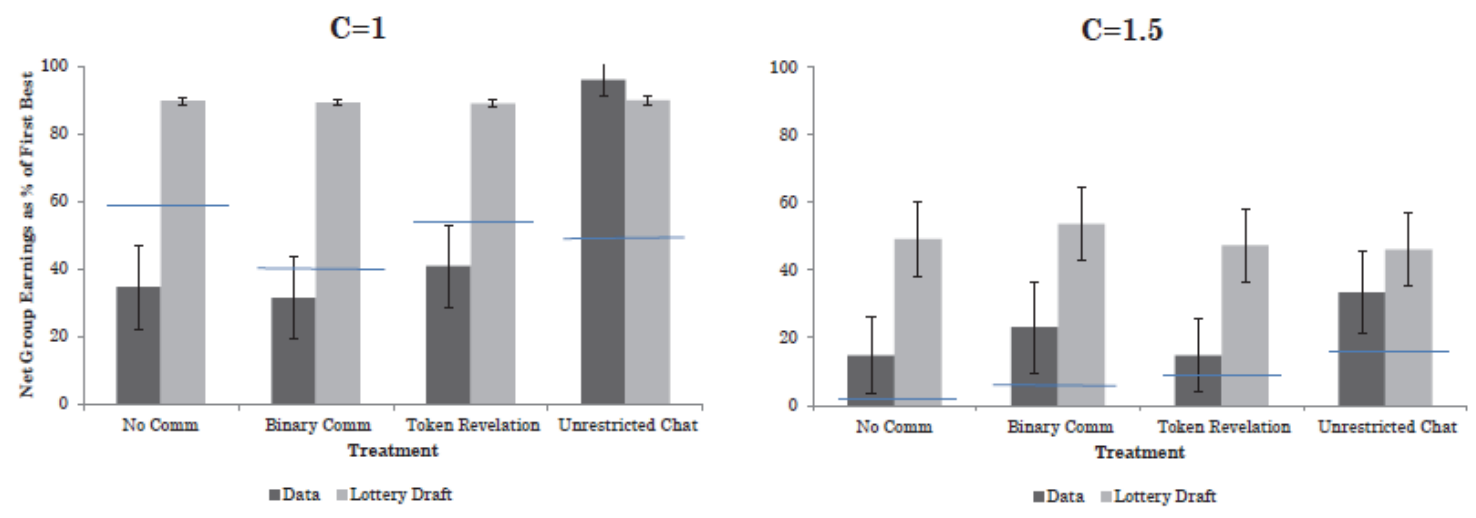

Figure 1: Normalized efficiency net of token values by treatments (rounds 11-20). Also shown are the 95\% confidence intervals. The horizontal line denotes the Bayes-Nash equilibrium normalized efficiency for each treatment. Lottery draft indicates the theoretical efficiency of the optimal communication equilibrium of the game, computed using the actual cost draws.

\subsection{Individual Behavior: Messaging and Contribution Strategies}

In this section, we take a deeper look at the individual choice data as a function of individual token values. We describe several findings about the strategies used by subjects in the communication stage, and about how the messages in the communication stage affected contribution decisions. These are presented below, separately for each of the communication structures. We use data from the last 10 rounds in this section. ${ }^{21}$

\footnotetext{
${ }^{20}$ Interestingly, in the 'chat' with $C=1$, groups do even slightly better than the optimal communication mechanism (lottery draft) and are close to the first-best outcome.

${ }^{21}$ For $C=1.5$, unless otherwise noted the observations with token values higher than 99 are dropped in this section because these individuals have a dominant strategy not to contribute, and almost never do. Across all communication
} 


\section{Binary Communication}

We start by analyzing the decision of whether to send an intent to spend message or an intent to keep message, and to compare this with the theoretical communication equilibrium given in Table 1. Theoretically, an intent to spend message should be observed $61 \%$ of the time in the $C=1$ treatment and $72 \%$ of the time in the $C=1.5$ treatment (conditional on token values less than 100). The observed values were $66 \%$ and $81 \%$, respectively. Thus, intent to spend messages were observed more frequently in the $C=1.5$ treatment, but in both cases such messages were observed more frequently than in the communication equilibrium given in Table 1. In both treatments, the frequency of intent to spend messages was decreasing in token value, as shown in the probit regression results displayed in Table 7.

\begin{tabular}{ccc}
\hline \hline & $\underline{C=1}$ & $\underline{C=1.5}$ \\
\hline Tokenvalue & $-0.005^{* * *}(0.002)$ & $-0.004^{* *}(0.002)$ \\
Constant & $1.11^{* * *}(0.32)$ & $1.61^{* * *}(0.40)$ \\
\hline Number of observations & 240 & 159 \\
Pseudo $\mathrm{R}^{2}$ & 0.061 & 0.056 \\
\hline \hline
\end{tabular}

* significant at $10 \%$;* significant at $5 \%$; ${ }^{* *}$ significant at $1 \%$

Table 7: Message behavior in the 'Binary Communication' treatment: Marginal effects from a probit regression with "message" as the dependent variable. Standard errors, clustered at the subject level, are in parentheses.

Next, we look at the effect of the binary messages on the spending decision of a subject. Table 8 shows the effect of own binary message and the profile of binary messages of the other two members of the group on own contribution decision. Because the messages are sent anonymously, the decision only depends on the number of other "intend to spend" messages in the group. The entries in the table are the contribution rates of the subject in each category. Based on the theoretical communication equilibrium in Table 1, when $C=1$, an individual is expected to contribute $74 \%$ of the time after she said that she intended to contribute in the message stage, while this number is $65 \%$ for $C=1.5$ sessions. When $C$ was 1 , an individual contributed $55 \%$ of the time after she said that she intended to contribute in the message stage, while this number was $66 \%$ for $C=1.5$ sessions. In contrast, individuals are expected to never contribute when they send an intent to keep message. Only $20 \%$ of the time did a person contribute after sending an intent to keep message in the $C=1$ sessions, while the comparable percentage for $C=1.5$ groups was $19 \%$.

The main departure from the theoretical conditional contribution rates, is that in theory, if you

treatments, in 632 cases where an individual's token value was greater than 99, there were only 11 observations of contribution. 


\begin{tabular}{lccc}
\hline \multirow{2}{*}{ Message sent by me } & \multicolumn{4}{c}{ Number of others saying "I intend to spend" } & \\
\cline { 2 - 4 } & & 1 & 2 \\
\hline & & $\underline{C=1}$ & \\
"I intend to spend" & $0.41(22)$ & $0.60(48)$ & $0.56(90)$ \\
"I intend to keep" & $0.25(12)$ & $0.23(44)$ & $0.13(24)$ \\
& & $\underline{C=1.5}$ & \\
"I intend to spend" & $0.38(21)$ & $0.82(49)$ & $0.62(58)$ \\
"I intend to keep" & $0.00(1)$ & $0.16(19)$ & $0.27(11)$ \\
\hline
\end{tabular}

Table 8: Fraction of times spent as a function of binary message profile. Total observations in parentheses.

and exactly one other individual sends a spend message then your predicted contribution rate is $100 \%$. This is not what we observe in the data, especially in the $C=1$ data. In the $C=1$ sessions, out of 48 observations with "I intend to spend my token" with exactly one other member sending this message, only 29 contribute. When no other member sent that message, only 9 of 22 contributed, but in the communication equilibrium none of these should be contributing. When both other members also reported that they intended to spend, 49 out of 90 contributed (54\%), while in theory this is predicted to be somewhat greater $(70 \%)$. For $C=1.5$ sessions, only $38 \%$ of subjects who reported an intent to spend carry out that intent when nobody else in the group sent the spend message. This contrasts with much higher rates when at least one other member says they intend to spend: $82 \%$ with one other and $62 \%$ with two others.

While these conditional contribution rates are different from the theoretical ones implied by Table 1, they reveal some interesting patterns in the contribution rates of individual who report an intent to spend. First, individuals are always more likely than not to carry out a reported intent to spend only if at least one other member of the group also reports an intent to spend. Second, this effect is strongest when exactly one other member reports an intent to spend. These patterns provide some additional insight into the earlier finding that binary communication partially alleviates the coordination failure that occurs when exactly one individual contribute (see Tables 4 and 5).

Individuals who report an intent to keep are always much more likely to keep than spend, regardless of how many other members of the group report an intent to spend. In cases where an individual reports "intend to keep", the effect of other messages on contribution decisions is small, and with no clear pattern. In the $C=1$ treatment, the probability of contributing declines very slightly with the number of other members who intend to spend, but it goes the other direction in the $C=1.5$ treatment.

To evaluate the statistical significance of these effects, Table 9 shows the results of a probit regression with contribution decision as the binary dependent variable, as well as whether the subject sent the message "I intend to spend my token" $\left(m_{i}=1\right)$ or "I intend to keep my token" $\left(m_{i}=0\right)$. 
We denote the number of other members in a group saying "I intend to spend" in the message stage as $M_{-i}$. The independent variables are as follows: own token value (tokenvalue $\left.i\right), \mathbb{1}\left\{M_{-i}=1\right\}$ : a binary variable indicating whether exactly one of the other two members sent the message "I intend to spend" and $\mathbb{1}\left\{M_{-i}=2\right\}$ : a binary variable indicating whether both the other members sent the message "I intend to spend".

The probit results in Table 9 confirm the effects identified in Table 8. An individual who sent "I intend to spend"message $\left(m_{i}=1\right)$ is more likely to contribute if there was at least one other member who expressed the willingness to spend in the message stage. This effect is significant in three of four cases, and the effect is strongest if there was exactly one other person in the group who reported an intent to spend. In contrast, none of the $M_{-i}$ coefficients are significant in "I intend to keep" case $\left(m_{i}=0\right)$.

\begin{tabular}{ccccc}
\hline \hline & $m_{i}=0$ & $m_{i}=1$ & $m_{i}=0$ & $m_{i}=1$ \\
\hline & $C=1$ & $C=1$ & $\underline{C=1.5}$ & $\underline{C=1.5}$ \\
tokenvalue $_{i}$ & $-0.005^{* * *}(0.002)$ & $-0.01^{* * *}(0.003)$ & $-0.005(0.003)$ & $-0.005^{* * *}(0.002)$ \\
$\mathbb{1}\left\{M_{-i}=1\right\}$ & $-0.01(0.14)$ & $0.26^{*}(0.15)$ & $-0.037(0.13)$ & $0.23^{* *}(0.1)$ \\
$\mathbb{1}\left\{M_{-i}=2\right\}$ & $-0.16(0.11)$ & $0.27^{*}(0.16)$ & $0.41(0.3)$ & $0.06(0.1)$ \\
Constant & $0.52(0.66)$ & $1.02^{* * *}(0.37)$ & $0.23(0.94)$ & $0.82^{* *}(0.34)$ \\
\hline Number of observations & 80 & 160 & 31 & 128 \\
Pseudo R $^{2}$ & 0.158 & 0.262 & 0.238 & 0.108 \\
\hline \hline
\end{tabular}

* significant at $10 \%$;* significant at $5 \%$;** significant at $1 \%$

Table 9: Decision in the Binary Communication treatment: Marginal effects from probit regression with "contribution decision" as the dependent variable, evaluated at the means of the independent variables. Standard errors, clustered at the subject level, are in parentheses.

\section{Token Revelation}

In the 'token revelation' treatment, the option of sending an empty message $\left(m_{i}=\right.$ ?) was chosen $7.5 \%$ of the time in $C=1$ groups and $14 \%$ of the time when $C$ was 1.5 . In $C=1$ groups, $49 \%$ of the reports were truthful and $41 \%$ of the reports were lower than the true token values, while individuals over-reported their token values only $10 \%$ of the time. A similar pattern was observed in the $C=1.5$ groups, where $44 \%$ of the reports were truthful, $38 \%$ were under-reported and $18 \%$ over-reported. Again, from the prevalence of under-reporting of token values it seems that individuals were trying to signal their group members that they can expect a contribution, perhaps believing that their own low report would induce others to contribute. In the $C=1.5$ sessions, individuals with token values greater than 100 under-reported $55 \%$ of the time while they reported truthfully around $35 \%$ of the time. This behavior is more difficult to rationalize, but again it's possible that subjects thought this 
would induce greater contribution by others. Out of 71 such instances when their token values were higher than 100, 36 times they sent a message less than 100. That appears to be one of the primary reasons of why efficiency is so low with direct mechanism communication when $C$ is 1.5. In contrast, out of 135 cases when the token value was less than 100, only $16 \%$ of the time individuals sent a message greater than 100 . They were truthful $48 \%$ of the time and over-reported $23 \%$ of the time.

Table 10 reports the results of a linear regression of the reported token value as a function of an individual's true token value ${ }^{22}$. As is evident, there is a significant positive relationship between true token values and reported token values. We ran a probit regression to analyze how the messages exchanged in the 'token revelation' round affect the contribution decision of a subject ${ }^{23}$. The dependent variable is the contribution decision which is binary: 1 if the individual contributes and 0 otherwise. The exogenous variables are (i) an individual's own tokenvalue $\left(\right.$ tokenvalue $\left._{i}\right)$, and (ii) $\mathbb{1}\left\{\right.$ tokenvalue $_{i}>$ $\left.\max \left\{m_{j}\right\}_{j \neq i}\right\}$ : a binary variable indicating whether or not the subject's token value is greater than the messages sent by other two members. We expect the sign of the coefficient on the variable $\mathbb{1}\left\{\right.$ tokenvalue $\left._{i}>\max \left\{m_{j}\right\}_{j \neq i}\right\}$ to be negative, meaning that an individual is less likely to contribute if her tokenvalue is higher than the other two members' reports. The coefficient is negative only for $C=1$ groups, and significant only at the $10 \%$ level (see Table 11). These findings, coupled with the fact that there is widespread mis-reporting of token values are suggestive of why groups are unable to use the ‘token revelation' stage for much gain in efficiency.

\begin{tabular}{ccc}
\hline \hline & $\underline{C=1}$ & $\underline{C=1.5}$ \\
\hline Tokenvalue & $0.38^{* *}(0.07)$ & $0.82^{* *}(0.12)$ \\
Constant & $17.18^{* * *}(3.79)$ & $13.10^{* * *}(6.75)$ \\
\hline Number of observations & 222 & 135 \\
$\mathrm{R}^{2}$ & 0.104 & 0.110 \\
\hline \hline
\end{tabular}

* significant at $10 \% ; * *$ significant at $5 \% ; * * *$ significant at $1 \%$

Table 10: Message behavior in the direct mechanism communication treatment: Linear regression with reported token value as the dependent variable. Standard errors, clustered at the subject level, are in parentheses.

\section{Chat}

We investigate the question of how pre-play communication in the form of unrestricted text chat helps subjects achieve higher payoffs. Table 12 gives the details of how many times a group reached some form of an agreement about the possible profile of actions to be taken by the members in the group

\footnotetext{
${ }^{22}$ The few observations where a subject did not send a message are dropped from the regression.

${ }^{23}$ The observations where at least one member of a group did not send any message are dropped.
} 


\begin{tabular}{ccc}
\hline \hline & $\underline{C=1}$ & $\underline{C=1.5}$ \\
\hline Tokenvalue $\left.\left.^{*} \max _{j}\right\}_{j \neq i}\right\}$ & $-0.014^{* * *}(0.002)$ & $-0.013^{* * *}(0.003)$ \\
$\mathbb{1}\left\{\right.$ tokenvalue $_{i}>\max \left\{\mathrm{m}^{*}(0.107)\right.$ & $0.029(0.131)$ \\
Constant $^{*}$ & $1.90^{* * *}(0.21)$ & $1.71^{* * *}(0.32)$ \\
\hline Number of observations & 222 & 135 \\
Pseudo R $^{2}$ & 0.32 & 0.31 \\
\hline \hline
\end{tabular}

* significant at $10 \%$;* significant at $5 \% ; * * *$ significant at $1 \%$

Table 11: Decision in the direct mechanism communication treatment: Marginal effects from probit regression with contribution decision as the dependent variable, evaluated at the mean of independent variables. Standard errors, clustered at the subject level, are in parentheses.

in the contribution stage. ${ }^{24}$

Groups reached an agreement nearly $88 \%$ of the time in $C=1$ treatment, and these agreements were carried out $94 \%$ of the time (99 out of 105). The ability of groups to reach agreements and carry them out was not nearly as successful in the $C=1.5$ treatment. The percentage of groups who reached an agreement was only $58 \%$, which is significantly lower. Moreover, of those groups that reached agreement, only 38\% (35 out of 93) carried out the agreement. The qualitative nature of the agreements was also different across the cost treatments. In the $C=1.5$ treatment fewer than half the agreements (38 out of 93) were for exactly two of the group members to spend, the efficient outcome, and only 20 of these groups carried out that efficient agreement. Just as many groups agreed to the non-credible agreement for all three to contribute. Not surprisingly these agreements were carried out only $8 \%$ of the time ( 3 out of 38 ). The agreements in the $C=1$ treatment were much different. 98 out of 105 agreements were for exactly two contributors and this was carried out $94 \%$ of the time (92 out of 98). There were five agreements for all three to spend and, perhaps surprisingly, all of these agreements were carried out. The difference between the $C=1.5$ and $C=1$ treatments is even starker after subjects have gained experience. In rounds 11-20 of the $C=1$ treatment agreements were nearly always reached (56/60) and moreover all 56 agreements were for exactly two contributors, and only once did the agreement failed to be carried out. In rounds 11-20 of the $C=1.5$ groups, there was an increase in the number of agreements for exactly two contributors (from 12/80 to 26/80), but these agreements were carried out less than half the time, which is the same rate as in the early rounds. Otherwise there was no notable change with experience in group agreements for the $C=1.5$ groups.

A group agrees to either of the three action profiles: (i) all members will spend their token, (ii) two of the members will spend their tokens, or (iii) everybody in the group will keep their tokens. Table 12 shows that when a group decides that all members should keep their tokens, they carry out

\footnotetext{
${ }^{24}$ Here we present data for all rounds, because there are interesting comparisons between early and late rounds.
} 


\begin{tabular}{cc|cccc}
\hline \hline Rounds & Agreements & & Agreed & Number & Spending \\
\hline$\underline{C=1}$ & & 0 & 1 & 2 & 3 \\
$1-10$ & $49(60)$ & $2(2)$ & $0(0)$ & $37(42)$ & $5(5)$ \\
$11-20$ & $56(60)$ & $0(0)$ & $0(0)$ & $55(56)$ & $0(0)$ \\
All rounds & $105(120)$ & $2(2)$ & $0(0)$ & $92(98)$ & $5(5)$ \\
& & & & & \\
$\underline{C}=1.5$ & & & & & \\
$1-10$ & $39(80)$ & $4(5)$ & $1(3)$ & $6(12)$ & $0(19)$ \\
$11-20$ & $54(80)$ & $6(6)$ & $1(3)$ & $14(26)$ & $3(19)$ \\
All rounds & $93(160)$ & $10(11)$ & $2(6)$ & $20(38)$ & $3(38)$ \\
\hline
\end{tabular}

Table 12: Agreement on an action profile in the communication stage: the first column gives the frequency of times a group reaches an agreement. Columns 2-5 gives the number of times the group members carry out the agreed action profile in the contribution stage (number of observations in parentheses).

this agreement quite well, almost $100 \%$ of the time, but when everyone in a group decides to spend their tokens, then only in a few number of cases they carry out this action. Of course, this is expected as a member might think that she could just keep her token while the other two are going to spend. This thinking actually leads to none or only one person contributing.

To summarize, the most striking feature of the chat communication is that when a group reaches an agreement in the $C=1$ sessions in rounds 11-20, 100\% of the time they decide that exactly 2 members will spend their tokens and they nearly always carry out this agreed upon profile of actions. This explains the extremely high efficiency levels reached in rounds 11-20 in $C=1$ sessions. This degree of success is not even close to being achieved by the $C=1.5$ groups, although the frequency of groups agreeing to profiles where exactly two members spend their tokens is higher in rounds 11-20, which accounts for the slightly higher efficiency in rounds 11-20 than the first ten rounds of play in the $C=1.5$ groups. $^{25}$

\section{Content Analysis of Chat Messages}

Finally, we provide a content analysis of the discussion in the communication round of the chat sessions to give a more complete picture of how chat facilitated cooperation and coordination. Each message sent by a subject was coded into one of the nine mutually exclusive categories, as enumerated in Table 13. The table also contains some verbatim examples of sentences/messages that fall under each

\footnotetext{
${ }^{25}$ Table 15 in the appendix compares the efficiency differences in the first ten and last ten rounds.
} 


\begin{tabular}{|c|c|}
\hline Code category & Examples \\
\hline Confirmation & okay; cool; yes; alright; done; great; yep \\
\hline Own token value & $\begin{array}{l}\text { token value is } 39 ; \text { I have a really high } \\
\text { value; its so low }\end{array}$ \\
\hline Others' token value or plan of action & $\begin{array}{l}\text { what is your token value } 3 \text { ?; are you going } \\
\text { to spend } 2 \text { ? }\end{array}$ \\
\hline $\begin{array}{l}\text { Strategy suggestion about others/own de- } \\
\text { cision/group decision }\end{array}$ & $\begin{array}{l}\text { I should keep my token; } 1 \text { and } 2 \text { should } \\
\text { spend and } 3 \text { keep; everyone should spend; } \\
\text { can I keep? }\end{array}$ \\
\hline $\begin{array}{l}\text { Informative/explaining something to } \\
\text { group members but not any strategy } \\
\text { suggestion }\end{array}$ & $\begin{array}{l}\text { if } 2 \text { spend then they both get } 100 \text {; token } \\
\text { values can never be higher than } 100\end{array}$ \\
\hline I plan to spend & spending; I will spend \\
\hline I plan to keep & keeping; I will keep \\
\hline $\begin{array}{l}\text { Conditional statement or ambigu- } \\
\text { ous/contradictory statement }\end{array}$ & $\begin{array}{l}\text { I will spend if someone else spends; I will } \\
\text { keep if you two spend; "I will keep" then } \\
\text { later on says "I will spend"; I will spend } \\
\text { or keep }\end{array}$ \\
\hline Irrelevant/Junk & lol; hehehe \\
\hline
\end{tabular}

Table 13: Content analysis: code categories.

category that were used in our experiments. The instructions did not indicate that the chat was to be in English, and subjects communicated in a language that was closer to SMS texts than to ordinary English.

Table 14 gives the percentage of all messages that fall in these nine categories in all four sessions of the 'chat' treatment. First notice that purely informative comments are infrequent, which one would expect if subjects understand the task. In general there was essentially no evidence of fundamental confusion. Second, while "confirmation" / "strategy suggestion" / "revelation of own token value" were used a lot in the $C=1$ sessions, chat messages are more evenly distributed across the various categories in the $C=1.5$ sessions, including somewhat more contradictory/ambiguous, junk and informative statements that were not related to coordinating on a strategy. There were also $50 \%$ more "plan to spend" messages in the $C=1.5$ sessions than in the $C=1$ sessions, and subjects often reneged on these announced plans in the second stage of the game. Messages falling in categories "strategy suggestion" and "confirmation" were used less in sessions with $C=1.5$ than in the $C=1$ sessions. Table 14 also shows that individuals used conditional statements or ambiguous messages very rarely in all of the sessions. Lastly, there was also no notable difference in the messages sent over time.

Thus, we see that revealing one's own token value coupled with a strategy suggestion about one's own as well as the entire group's strategies helps in achieving "efficient" outcomes in the situation when it is common knowledge that everyone has costs less than the 'public' benefit. However, in the other situation, there is a lack of use of these message categories and efficiency is also not as high. Apart from this, a closer look at the transcript of the text chat reveals that in the $C=1.5$ sessions 


\begin{tabular}{|c|c|c|}
\hline Code category & $C=1$ & $C=1.5$ \\
\hline Confirmation & 16.8 & 5.8 \\
\hline Own token value & 24.3 & 12.6 \\
\hline Others' token value or plan of action & 5.8 & 11.1 \\
\hline $\begin{array}{l}\text { Strategy suggestion about others/own de- } \\
\text { cision/group decision }\end{array}$ & 16.2 & 6.4 \\
\hline $\begin{array}{l}\text { Informative/explaining something to } \\
\text { group members but not any strategy } \\
\text { suggestion }\end{array}$ & 6.9 & 19.2 \\
\hline I plan to spend & 11.7 & 17.4 \\
\hline I plan to keep & 4.4 & 8.6 \\
\hline $\begin{array}{l}\text { Conditional statement or ambigu- } \\
\text { ous/contradictory statement }\end{array}$ & 1.5 & 3.3 \\
\hline Irrelevant/Junk & 12.4 & 15.6 \\
\hline
\end{tabular}

Table 14: Content analysis: percentage of messages falling in the code categories.

there is a lot of discussion regarding lying, trust and promises, whereas, none of these terms are used when $C=1$. So, in the $C=1.5$ sessions, there is an atmosphere of mistrust which is certainly a reason as to why subjects do not do as well in these sessions even with unrestricted text chat. A quote from one of the texts from a subject aptly summarizes the situation: "this test really shows how low humans have fallen".

\section{Conclusion}

We investigated the effect on efficiency, in a public goods game where individual endowments are private information, of non-binding pre-play communication structures differing in the richness of the message space. Neither the one-time simultaneous exchange of binary messages meant to reveal one's intention to contribute nor the one-time numerical report of private information is enough to create a significant efficiency gain relative to the situation without any form of pre-play communication. It is only when participants are provided with the opportunity to engage in text chat in an unrestricted fashion do we find that efficiency and public good provision are significantly higher.

The theoretical predictions coming out of mechanism design theory provide only theoretical upper bounds on efficiency, similar to how repeated game theory provides only theoretical upper bounds on subgame perfect equilibrium payoffs in repeated games. In two of the communication treatments, these bounds were not achieved, which suggests that in order to implement the optimal incentive compatible allocation through unmediated cheap talk, as opposed to a formal mechanism, one probably needs a richer communication language than simply binary or direct messages.

Without a 'common language' there is no obvious way to interpret the binary or the numerical messages among subjects in a way that can be easily translated into coordinated actions. The natural 
language communication is successful even though the groups are rotated every round, so the main positive finding is not due to repeated game effects. Unrestricted chat gives the subjects the opportunity to understand and interpret each others' intentions and messages and make credible commitments that they are willing to carry out.

A second finding is that the gains relative to the situation with no communication are much higher when it is common knowledge that everyone has costs less than the 'public' benefit. This is predicted by the theoretical analysis based on mechanism design, and is intuitive because in this situation the question is not whether the good should be provided but rather which of the two people in a group are going to provide it. In contrast, when some individuals have higher costs than the public benefit, individual rationality constraints impose severe restrictions on the gains from communication. In this case a group must first establish whether it is feasible for the good to be voluntarily provided, which exacerbates both the free rider problem and the incentive for individuals to truthfully reveal their private information. As a result there is more severe mis-coordination and mis-representation.

This study raises a number of questions that suggest interesting possibilities for follow-up studies as future research. In contrasting the weak performance of preplay communication in the binary and token communication treatments with the more successful performance of natural language chat communication, we have highlighted the fact that natural language is much richer. However, it is richer in a variety of different ways that could be further investigated in terms of these different components. For example, the natural language chat is sequential, and group members have an opportunity to react and respond to messages sent by other members. It is possible that the other two communication protocols would be more successful at achieving coordination if communication were sequentially ordered or if individuals could send messages repeatedly over several stages. ${ }^{26}$ Alternatively, one might conjecture that the natural language chat might not perform as well if each group member could only send one message during the cheap talk phase. These possibilities and others indicate a range of possible variations on our experimental design that are worth exploring.

Finally, as a methodological note, this paper provides an example of how mechanism design theory can be usefully applied as a framework for understanding data from laboratory experiments of games with pre-play communication. The theoretical justification for this framework is based on the mechanism design approach to communication in games (Myerson 1986, Forges 1986), an approach that has promising applications to a wide range of other kinds of experiments, such as committee deliberation (Goeree and Yariv 2011), face-to-face bargaining, and collusion in auctions.

\footnotetext{
${ }^{26}$ Cooper et al. (1989) report such a finding for battle of the sexes games.
} 


\section{References}

[1] Agastya, M., Menezes, F. and Sengupta, K. (2007), "Cheap talk, efficiency and egalitarian cost sharing in joint projects," Games and Economic Behavior, 60, 1-19.

[2] Bagnoli, M. and McKee, M. (1991), "Voluntary contribution games: efficient private provision of public goods," Economic Inquiry, 29(2), 351-366.

[3] Belianin, A. and Novarese, M. (2005), "Trust, communication and equilibrium behavior in public goods," Working paper.

[4] Bochet, O., Page, T. and Putterman, L. (2006), "Communication and punishment in voluntary contribution experiments," Journal of Economic Behavior and Organization, 60, 11-26.

[5] Brosig, J., Ockenfels, A. and Weimann, J. (2003), "The effect of communication media on cooperation," German Economic Review, 4, 217-242.

[6] Cason, T. and Khan, F. (1999), "A laboratory study of voluntary public goods provision with imperfect monitoring and communication," Journal of Development Economics, 58, 533-552.

[7] Cooper, R., DeJong, D., Forsythe, R. and Ross, T. (1989), "Communication in the Battle of the Sexes Game: Some Experimental Results," Rand Journal of Economics, 20(4), 568-587.

[8] Cooper, R., DeJong, D., Forsythe, R. and Ross, T. (1992), "Communication in coordination games," Quarterly Journal of Economics, 107, 739-771.

[9] Costa, F. J. and Moreira, H. A. (2012), "On the limits of cheap talk for public good provision," Working paper.

[10] Crawford, V. P. and Sobel, J. (1982), "Strategic information transmission," Econometrica, 50(6), 1431-1451.

[11] Dawes, R. M., Orbell, J. M., Simmons, R. T. and Van de Kragt, A. (1986), "Organizing groups for collective action," American Political Science Review, 80(4), 1171-1185.

[12] Ferejohn, F., Forsythe, R., Noll, R. and Palfrey, T. R. (1982), "An experimental examination of auction mechanisms for discrete public goods," in V. Smith ed. Research in Experimental Economics, Vol 2, 175-199, JAI Press.

[13] Forges, F. (1986), “An approach to communication equilibria,” Econometrica, 54, 1375-1386. 
[14] Goeree, J. and C. Holt (2005), "An Explanation of Anomalous Behavior in Models of Political Participation," American Political Science Review, 99(2), 201-213.

[15] Goeree, J. and L. Yariv (2011), “An Experimental Study of Collective Deliberation” Econometrica, 79(3), 893921.

[16] Isaac, R. M. and Walker, J. M. (1988), "Communication and free-riding behavior: the voluntary contribution mechanism," Economic Inquiry, 264, 585-608.

[17] Kawamura, K. (2011), "A model of public consultation: why is binary communication so common?," The Economic Journal, 121, 819-842.

[18] Ledyard, J. (1995), "Public goods: a survey of experimental research," in J. Kagel and A. Roth eds. Handbook of Experimental Economics, Princeton: Princeton University Press.

[19] Ledyard, J. and Palfrey, T. R. (2002), "The approximation of efficient public good mechanisms by simple voting schemes," Journal of Public Economics, 83, 153-171.

[20] Myerson, R. (1986), "Multistage games with communication," Econometrica, 54, 323-358.

[21] Offerman, T., A. Schram, and J. Sonnemans (1998), "Quantal response models in step-level public good games" European Journal of Political Economy, 14(1), 89-100.

[22] Ostrom, E. and Walker, J. M. (1991), "Communication in a commmons: cooperation without external enforcement," in T. R. Palfrey, ed. Laboratory Research in Political Economy, Ann Arbor: University of Michigan Press.

[23] Palfrey, T. R. and Rosenthal, H. (1991), "Testing for effects of cheap talk in a public goods game with private information," Games and Economic Behavior, 3, 183-220.

[24] Smith, V. (1980), "Experiments with a decentralized mechanism for public good decisions," American Economic Review, 70, 584-599.

[25] Van de Kragt, A., Orbell, J. and Dawes, R. (1983), "The minimal contributing set as a solution to public goods problems," American Political Science Review, 77, 112-121. 


\section{APPENDICES}

\section{A Proofs of Propositions 1 and 2}

\section{Low Cost Distribution $(C \leq 1)$}

Proposition 1. If costs are uniformly distributed on $[0,1]$, then the solution to (2) is $P(c)=1$ and $A(c)=\frac{K}{N}$ for all $c \in[0,1]$.

Proof. We prove a more general version of this proposition for any admissible $F$. Denoting $U(c)=$ $P(c)-c A(c)$, we have, from incentive compatibility that in any differentiable mechanism we must have $U^{\prime}(c)=-A(c) \geq 0$ and $U^{\prime \prime}(c)=-A^{\prime}(c) \geq 0$, and differentiability of $U$ and $A$ holds almost everywhere in $[0,1]$. Hence we can rewrite the optimization problem as:

$$
\begin{aligned}
& \max _{(P, A)} \int_{0}^{C} U(c) f(c) d c \\
& \text { subject to } \\
& A(c)=-U^{\prime}(c) \forall c \\
& U^{\prime \prime}(c) \geq 0 \forall c \\
& \frac{K}{N} \int_{0}^{C} P(c) f(c) d c \leq \int_{0}^{C} A(c) f(c) d c \\
& 0 \leq P(c) \leq 1 \forall c, 0 \leq A(c) \leq 1 \forall c
\end{aligned}
$$

The first incentive constraint, $A(c)=-U^{\prime}(c)$ implies we can write

$$
U(c)=U(0)-\int_{0}^{c} A(x) d x
$$

We can rewrite (9) as

$$
\begin{aligned}
& \max _{(P, A)} U(0)-\int_{0}^{C}\left(\int_{0}^{c} A(x) d x\right) f(c) d c \\
& \text { subject to } \\
U^{\prime \prime}(c) \geq & 0 \forall c \\
\frac{K}{N} \int_{0}^{C} P(c) f(c) d c \leq & \int_{0}^{C} A(c) f(c) d c \\
0 \leq & P(c) \leq 1 \forall c, 0 \leq A(c) \leq 1 \forall c
\end{aligned}
$$


which is equivalent to:

$$
\begin{aligned}
& \max _{(P, A)} U(0)-\int_{0}^{C} A(c) d c+\int_{0}^{C} F(c) A(c) d c \\
\text { subject to } & \\
U^{\prime \prime}(c) \geq & 0 \forall c \\
\frac{K}{N} \int_{0}^{C} P(c) f(c) d c \leq & \int_{0}^{C} A(c) f(c) d c \\
0 \leq & P(c) \leq 1 \forall c, 0 \leq A(c) \leq 1 \forall c
\end{aligned}
$$

Let $\left(P^{*}, A^{*}\right)$ be a solution to $(11)$, denote $\bar{A}=\int_{0}^{C} A^{*}(c) d c$, and note that $U^{*}(0)=P^{*}(0)-A^{*}(0)$. Whatever the solution, it must be that $A^{*}(\cdot)$ solves:

$$
\begin{aligned}
& \max _{A(\cdot)} \int_{0}^{C} F(c) A(c) d c \\
& \text { subject to } \\
& A(0)=A^{*}(0) \\
& A^{\prime}(c) \leq 0 \forall c \\
& \int_{0}^{C} A(c) d c=\bar{A} \\
& 0 \leq A(c) \leq 1 \forall c
\end{aligned}
$$

Because $F$ is non-negative and strictly increasing and $A$ is required to be non-increasing, this immediately implies that $A^{* \prime}=0$ and hence $A^{*}(c)=\bar{A}$ for all $c$. Because $A^{*}$ is flat, incentive compatibility implies that $P^{*}$ is also flat, with $P^{*}(c)=P^{*}(0)$ for all $c$. Furthermore, it must be that $\bar{A}^{*}$ is as small as possible, subject to the feasibility constraint, implying immediately that $\frac{K}{N} \int_{0}^{C} P^{*}(c) f(c) d c=\int_{0}^{C} A^{*}(c) f(c) d c$. Hence, the problem reduces to finding the optimal flat mechanism that is also budget balanced, which trivially implies $P^{*}(c)=1$ for all $c$ and $A^{*}(c)=\frac{K}{N} \forall c$. In other words, the public good is always provided, and exactly $K$ individuals are randomly selected to contribute. Observe that ex post individual rationality is satisfied and also that implementability of the reduced form mechanism is trivial.Thus, the ex ante efficient mechanism does not use any information about types. It completely solves the coordination problem of over- and under-contribution, and the free rider problem but is completely unable to resolve the informational source of cost inefficiency which would require low cost types to contribute more often than high cost types. 
High cost distribution: $C=1.5$

Proposition 2. If costs are uniformly distributed on $[0,1.5], K=2$, and $N=3$, then the solution to (3) is given by:

$$
\begin{aligned}
c^{*} & =0.75 \\
P(c) & =0.75 \forall c \in\left[0, c^{*}\right] \\
& =0.25 \forall c \in\left[c^{*}, 1.5\right] \\
A(c) & =0.67 \forall c \in\left[0, c^{*}\right] \\
& =0 \forall c \in\left[c^{*}, 1.5\right]
\end{aligned}
$$

Proof. As for Proposition 2, we provide a more general proof for the case where $K, N$ are arbitrary integers with $1 \leq K \leq N$.

Because IC implies $A(c)=-U^{\prime}(c) \forall c$, we have:

$$
\begin{aligned}
U(c) & =U(1.5)+\int_{c}^{1.5} A(x) d x \\
& =\underline{P}+\int_{c}^{\max \left\{c, c^{*}\right\}} \widehat{A}(x) d x
\end{aligned}
$$

so the optimization problem can be rewritten as:

$$
\begin{aligned}
& \max _{\left(\widehat{P}, \widehat{A}, \underline{P}, c^{*}\right)} \underline{P}+\int_{0}^{1.5}\left(\int_{c}^{\max \left\{c, c^{*}\right\}} \widehat{A}(x) d x\right) \frac{1}{1.5} d c \\
& \text { subject to } U^{\prime \prime}(c) \geq 0 \forall c \\
&\left.\frac{K}{N}\left\{\underline{P}\left[1-\frac{c^{*}}{1.5}\right)\right]+\int_{0}^{c^{*}} \widehat{P}(c) \frac{1}{1.5} d c\right\} \leq \int_{0}^{c^{*}} \widehat{A}(c) \frac{1}{1.5} d c \\
& 0 \leq \widehat{P}(c) \leq \underline{P} \forall c, 0 \leq \widehat{A}(c) \leq 1 \forall c \in\left[0, c^{*}\right]
\end{aligned}
$$


where $\frac{1}{1.5}$ is just the density function of the cost distribution. This is equivalent to:

$$
\begin{aligned}
& \max _{\left(\widehat{P}, \widehat{A}, \underline{P}, c^{*}\right)} \underline{P}+\int_{0}^{c^{*}} \frac{c}{1.5} \widehat{A}(c) d c \\
& \text { subject to } \\
U^{\prime \prime}(c) \geq & 0 \forall c \\
\frac{K}{N}\left\{\underline{P}\left[1-\frac{c^{*}}{1.5}\right]+\int_{0}^{c^{*}} \widehat{P}(c) \frac{1}{1.5} d c\right\} \leq & \int_{0}^{c^{*}} \widehat{A}(c) \frac{1}{1.5} d c \\
0 \leq & \widehat{P}(c) \leq \underline{P} \forall c, 0 \leq \widehat{A}(c) \leq 1 \forall c \in\left[0, c^{*}\right]
\end{aligned}
$$

Let $\left(\widehat{P}^{*}, \widehat{A}^{*}, \underline{P}^{*}, c^{*}\right)$ be a solution to $(13)$, denote $\bar{A}=\int_{0}^{c^{*}} \widehat{A}(c) \frac{1}{1.5} d c$. If this is optimal, then it must be that the budget constraint holds with equality. That is:

$$
\frac{K}{N}\left\{\underline{P}^{*}\left[1-\frac{c^{*}}{1.5}\right]+\int_{0}^{c^{*}} \widehat{P}^{*}(c) \frac{1}{1.5} d c\right\}=\bar{A}^{*}
$$

If not, $\underline{P}$ can be increased without violating any constraints and increases the objective function, $\underline{P}+\int_{0}^{c^{*}} \frac{c}{1.5} \widehat{A}(c) d c$. This implies that whenever the public good is produced, it is produced with exactly $K$ contributions. Second, as in the case studied earlier, with $C \leq 1$, for any $\bar{A}, \widehat{A}^{*}(c)$ must solve:

$$
\begin{aligned}
\max _{\widehat{A}(\cdot)} \int_{0}^{c^{*}} \frac{c}{1.5} \widehat{A}(c) d c & \text { subject to } \\
\widehat{A}^{\prime}(c) \leq & 0 \forall c \in\left[0, c^{*}\right] \\
\int_{0}^{c^{*}} \widehat{A}(c) d c & \bar{A} \\
0 \leq & A(c) \leq 1 \forall c \in\left[0, c^{*}\right]
\end{aligned}
$$

Because $F$ is non-negative and strictly increasing and $\widehat{A}$ is required to be non-increasing, this immediately implies that $\widehat{A}^{* \prime}=0$ and hence $\widehat{A}^{*}(c)=\bar{A}^{*}$ for all $c \in\left[0, c^{*}\right]$. Because $\widehat{A}^{*}$ is flat, incentive compatibility implies that $\widehat{P}^{*}$ is also flat, with $\widehat{P}^{*}(c)=\bar{P}^{*}$ for all $c \in\left[0, c^{*}\right]$. Next observe that, given any $c^{*}, \underline{P}$ is maximized by producing the good whenever at least $K$ individuals have a cost $c \in\left[0, c^{*}\right]$. Budget balance then implies that whenever this is the case, the individuals share the cost equally. Hence the mechanism looks qualitatively very similar to the optimal mechanism when $C \leq 1$. In the direct version of this mechanism, everyone reports their cost; if at least $K$ individuals report a cost 
less than or equal to $c^{*}$, the public good is produced and exactly $K$ of these "low cost" individuals are randomly selected to contribute. Otherwise the public good is not produced at all. Finally, the critical cost, $c^{*}$, is pinned down by incentive constraint on the $c^{*}$ type, who must be indifferent between reporting $c^{*}$ with an expected payoff of $\bar{P}^{*}-c^{*} \bar{A}^{*}$ and reporting some higher cost and getting an expected payoff of $\underline{P}^{*}$. Thus, $c^{*}$ is characterized by:

$$
\bar{P}^{*}-c^{*} \bar{A}^{*}=\underline{P}^{*}
$$

Where, by the characterization above, we have:

$$
\begin{aligned}
\bar{P}^{*} & =\sum_{j=K-1}^{N-1}\left(\begin{array}{c}
N-1 \\
j
\end{array}\right)\left[\frac{c^{*}}{1.5}\right]^{j}\left[1-\frac{c^{*}}{1.5}\right]^{N-1-j} \\
\underline{P}^{*} & \left.=\sum_{j=K}^{N-1}\left(\begin{array}{c}
N-1 \\
j
\end{array}\right)\left[\frac{c^{*}}{1.5}\right)\right]^{j}\left[1-\frac{c^{*}}{1.5}\right]^{N-1-j} \\
\bar{A}^{*} & =\sum_{j=K-1}^{N-1} \frac{K}{j+1}\left(\begin{array}{c}
N-1 \\
j
\end{array}\right)^{N}\left[\frac{c^{*}}{1.5}\right]^{j}\left[\frac{c^{*}}{1.5}\right]^{N-1-j}
\end{aligned}
$$

If there are multiple values of $c^{*} \in[0,1)$ that solve $(14)$, then the optimal mechanism corresponds to the highest such value of $c^{*}$, because it leads to the highest possible value of $\underline{P} .{ }^{27}$

\section{Solution for experimental parameters: $K=2, N=3$}

Substituting into (15) the specific values $K=2, N=3$, and denoting $q=\frac{c^{*}}{1.5}$, we get

$$
\begin{aligned}
& \bar{P}^{*}=q^{2}+2 q(1-q) \\
& \underline{P}^{*}=q^{2} \\
& \bar{A}^{*}=\frac{2}{3} q^{2}+2 q(1-q)
\end{aligned}
$$

where $q=\frac{c^{*}}{1.5}$. Solving for $q$ using (14) gives $q=0.5$. Hence $c^{*}=0.75, \underline{P}^{*}=0.25, \bar{P}^{*}=0.75$ and $\bar{A}^{*}=\frac{2}{3}$. The ex ante probability the public good will be provided is 0.50 . This can also be implemented in the chat communication or in the token revelation communication protocols.

\footnotetext{
${ }^{27}$ We conjecture that the above proof extends to non-uniform distributions. For similar reasons to proposition 1 , the argument above probably goes through for any continuously differentiable distribution function $F$ on $[0, C]$, where $0<C \leq 1, F(0)=0$ and $F(C)=1$. However, the existence of the binding ex post individual rationality constraint lead to some technical problems that may require stronger assumptions about $F$.
} 


\section{B Sample Instructions (for $C=1$ )}

Thank you for agreeing to participate in this group decision making experiment. During the experiment we require your complete, undistracted attention, and ask that you follow instructions carefully. You should not open other applications on your computer, chat with other students, or engage in other distracting activities, such as using your phone, reading books, etc.

You will be paid for your participation in cash, at the end of the experiment. Different participants may earn different amounts. What you earn depends partly on your decisions, partly on the decisions of others, and partly on chance.

The entire experiment will take place through computer terminals, and all interaction between you will take place through the computers. It is important that you not talk or in any way try to communicate with other participants during the experiment, except as instructed.

We will start with a brief instruction period. During the instruction period, you will be given a complete description of the experiment and will be shown how to use the computers. If you have any questions during the instruction period, raise your hand and your question will be answered out loud so everyone can hear. If you have any questions after the experiment has begun, raise your hand, and an experimenter will come and assist you.

You will make choices over a sequence of 20 matches. In each match, you will be assigned to a group with two other participants in the room. In every match, you and the two other participants you are matched with each makes a single decision. Your earnings for that match will depend on all three group members' decisions, but are completely unaffected by the decisions made by participants assigned to other groups. We will explain exactly how these payoffs are computed in a minute.

At the end of the session, you will be paid the sum of what you have earned in all matches, plus the show-up fee of $\$ 5$. Everyone will be paid in private and you are under no obligation to tell others how much you earned. Your earnings during the experiment are denominated in points. At the end of the experiment you will be paid $\$ 1$ for every 100 points you have earned.

Every match proceeds as follows. At the beginning of each match, we randomly divide you into 3-member groups called committees. The committees are completely independent of each other and payoffs and decisions in one committee have no effect on payoffs and decisions in other committees. Each member has a single token and can either spend or keep that token. Each member is also assigned a private token value, which is equally likely to be any amount of points between 1 and 100 (150 in $C=1.5$ instructions). Token value assignments are completely independent across members, across committees, and across matches. Thus, your own token value tells you absolutely nothing about the token value of the other members, and has no effect on any future token values that will be assigned 
to you or anyone else.

Payoffs are computed as follows. If you keep your token you earn your token value in that match plus you earn 100 points if both other members of your committee decide to spend their tokens. If you choose to spend your token, then you earn 100 points if at least one other member of your group spends their token, and you earn 0 points if no other member of your group spends their token. This is summarized in the following table.

\begin{tabular}{|c|c|c|}
\hline Your Decision & \#Others Spending & Your Earnings \\
\hline \multirow{3}{*}{ SPEND } & 0 & 0 \\
\cline { 2 - 3 } & 1 & 100 \\
\cline { 2 - 3 } & 2 & 100 \\
\hline \multirow{3}{*}{ KEEP } & 0 & YOUR TOKEN VALUE \\
\cline { 2 - 3 } & 1 & YOUR TOKEN VALUE \\
\cline { 2 - 3 } & 2 & YOUR TOKEN VALUE + 100 \\
\hline
\end{tabular}

[The following paragraph only in the 'No Communication' treatments.] Every match you are prompted to make your choice to either keep or spend your token. When everyone has made a choice, the outcome and the choices of the other members of your committee are revealed, and this determines your earnings for the match.

[The following two paragraphs only in the 'Binary Communication' treatments.] Before anyone makes a spending decision, each of you will have an opportunity to give the other members of your committee some indication of what your spending decision might be. There are exactly two messages which you can send. They are:

\section{MESSAGE A: "I INTEND TO SPEND MY TOKEN"}

\section{MESSAGE B: "I INTEND TO KEEP MY TOKEN"}

Please remember that these are only messages and are not binding in any way. When the message stage ends, you are told the intent messages of the others in your committee and the decision stage begins wherein you are prompted to make your choice to either keep or spend your token. This decision is binding. When everyone has made a choice, the outcome and the choices of the other members of your committee are revealed, and this determines your earnings for the match.

[The following two paragraphs only in the 'Token Revelation' treatments.] Before anyone makes a spending decision, your committee has a 20 seconds message stage, during which you are allowed to 
send a message to the other members of your committee. This message can only be an integer between 1 and 100 (150 in $C=1.5$ instructions) and you are allowed to send only one such message. The integer value you send are seen by both other members of your committee. In the situation where you do not send any message, it will be shown as a "question mark" to other members of your committee at the end of the message stage.

When the message stage ends, the decision stage begins wherein you are prompted to make your choice to either keep or spend your token. When everyone has made a choice, the outcome and the choices of the other members of your committee are revealed, and this determines your earnings for the match.

[The following two paragraphs only in the 'Chat' treatments.] Before anyone makes a spending decision, your committee has a 60 seconds discussion period, during which you are allowed to send messages to the other members of your committee. The messages you send are seen by both other members of your committee. The messages must conform to the following rules: (1) Your messages must be relevant to the experiment. Do not engage in social chat or use emoticons. (2) You are not permitted to send messages intended to reveal your identity. (3) The use of threatening or offensive language, including profanity, is not permitted. (4) Do not send blank or nonsense messages.

When the discussion period ends, the decision stage begins wherein you are prompted to make your choice to either keep or spend your token. When everyone has made a choice, the outcome and the choices of the other members of your committee are revealed, and this determines your earnings for the match.

When all committees have finished the first match, we then go to the next match. You will be randomly re-matched into new 3-person committees and everyone is independently and randomly assigned a new token value between 1 and 100 (150 in $C=1.5$ instructions). Every match proceeds according to exactly the same rules as described above. ${ }^{28}$

We will now give you a chance to get used to the computers with a brief practice session. Are there any questions before we begin the practice match? [ANSWER QUESTIONS] You will not be paid for this practice match; it is just to get you familiar with the display. During the practice session, do not press any keys or click with your mouse, unless instructed to. When we instruct you, please do exactly as we ask.

Please pull out your dividers so we can begin the practice session. You are not paid for this practice match.

\footnotetext{
${ }^{28}$ The text of the instructions up to this point was distributed as a handout to each subject and they followed along as the experimenter read them aloud. The remaining script of the instructions, below, was only read aloud by the experimenter.
} 


\section{[SCREEN 3]}

When the match begins, you will see a screen on your computer that looks like this. You have a temporary committee ID that is printed at the top of the right half of your screen. This ID will either be 1,2 , or 3 . Your temporary ID will change randomly from match to match. Your private token value for this match is displayed just below this. The bottom window of your screen is the history table, which is blank now because nothing has happened yet.

[START GAME on SERVER]

Please double click on the icon on your desktop that says MultistageClient. When the computer prompts you for your name, type your First and Last name. Then click SUBMIT and wait for further instructions. Please make your decision. After you and other members in your committee make a decision, you will be shown the decision screen about your choice along with the choices of other members. You will also be shown how much you earned in the match.

[explain the utility of the HISTORY screen using Screen 4 display]

This ends the practice match. Are there any questions before we go to the first paid match?

[WAIT FOR QUESTIONS]

[SHOW SUMMARY SCREEN 5, WHICH SUMMARIZES THE BASIC RULES OF THE EXPERIMENT, AND READ IT. KEEP DISPLAYED DURING EXPERIMENT.]

We will now begin with the first of the 20 paid matches. Please pull out your dividers. If there are any problems or questions from this point on, raise your hand and I will come and assist you.

(Start match 1)

[Run matches 1-20]

This completes the experiment. Your total earnings for the 20 matches are displayed in a popup window. Please record this amount on your record sheet, and then click ok. You will be paid this amount plus your $\$ 5$ show-up fee.

[Experimenter: make sure they click ok so the output can be written and the pay file printed] Do not use the computers or talk with each other. We will pay each of you in private in the next room in the order of your seat numbers. Please sign and turn in your record sheet when you receive payment. You are under no obligation to reveal your earnings to the other participants. Thank you for your participation. 


\section{Additional Tables}

The following tables expand the analysis presented in the body of the paper, where applicable, to include data from the early rounds 1-10.

\begin{tabular}{|c|c|c|c|c|}
\hline Rounds & No Comm. & Binary Comm. & Token Revelation & Chat \\
\hline \multicolumn{5}{|c|}{$\underline{C=1}$} \\
\hline $1-10$ & $\begin{array}{l}113.2 \\
(15.7)\end{array}$ & $\begin{array}{c}89.7 \\
(14.0)\end{array}$ & $\begin{array}{c}95.8 \\
(14.8)\end{array}$ & $\begin{array}{c}176.8^{* *} \\
(11.9)\end{array}$ \\
\hline $11-20$ & $\begin{array}{c}88.0 \\
(15.3)\end{array}$ & $\begin{array}{c}80.2 \\
(14.7)\end{array}$ & $\begin{array}{l}101.5 \\
(14.6)\end{array}$ & $\begin{array}{c}221.1^{* * *} \\
(7.1)\end{array}$ \\
\hline All rounds & $\begin{array}{l}100.6 \\
(10.9)\end{array}$ & $\begin{array}{c}84.9 \\
(10.1)\end{array}$ & $\begin{array}{c}98.6 \\
(10.4)\end{array}$ & $\begin{array}{c}198.9 * * * \\
(7.2)\end{array}$ \\
\hline \multicolumn{5}{|c|}{$\underline{C=1.5}$} \\
\hline $1-10$ & $\begin{array}{c}67.0 \\
(14.0)\end{array}$ & $\begin{array}{c}82.0 \\
(13.8)\end{array}$ & $\begin{array}{c}55.1 \\
(13.8)\end{array}$ & $\begin{array}{c}48.5 \\
(12.4)\end{array}$ \\
\hline $11-20$ & $\begin{array}{c}39.6 \\
(12.4)\end{array}$ & $\begin{array}{c}58.7 \\
(13.4)\end{array}$ & $\begin{array}{c}42.1 \\
(11.9)\end{array}$ & $\begin{array}{c}83.4^{* * *} \\
(13.9)\end{array}$ \\
\hline All rounds & $\begin{array}{l}53.3 \\
(9.4)\end{array}$ & $\begin{array}{l}70.3 \\
(9.6)\end{array}$ & $\begin{array}{l}48.6 \\
(9.1)\end{array}$ & $\begin{array}{l}66.0 \\
(9.4)\end{array}$ \\
\hline
\end{tabular}

* significant at $10 \%$; ${ }^{* *}$ significant at $5 \%$; $* *$ significant at $1 \%$. These significance levels are for one-tailed Mann-Whitney tests of difference in the net earnings across the communication treatment and 'No Communication'.

Table 15: Average group earnings net of token values. Standard errors in parentheses. 


\begin{tabular}{ccccc}
\hline \hline Rounds & No Comm. & Binary Comm. & Token Revelation & Chat \\
\hline \multicolumn{5}{c}{$\underline{C=1}$} \\
1-10 & $54.3(70)$ & $47.5(80)$ & $48.8(80)$ & $85.0^{* * *}(60)$ \\
$11-20$ & $42.9(70)$ & $41.3(80)$ & $50.0(80)$ & $98.3^{* * *}(60)$ \\
All rounds & $48.6(140)$ & $44.4(160)$ & $49.4(160)$ & $91.7^{* * *}(120)$ \\
& & $\underline{C}=1.5$ & & \\
& & & & \\
$1-10$ & $38.8(80)$ & $43.8(80)$ & $38.8(80)$ & $31.3(80)$ \\
$11-20$ & $27.5(80)$ & $37.5(80)$ & $25.0(80)$ & $42.5^{* *}(80)$ \\
All rounds & $33.1(160)$ & $40.6(160)$ & $31.9(160)$ & $36.9(160)$ \\
\hline
\end{tabular}

$*$ significant at $10 \% ; * *$ significant at $5 \% ; * * *$ significant at $1 \%$. These significance levels are for difference in proportions z-tests in the public good provision across the communication treatment and 'No Communication'.

Table 16: Frequency of public good provision. Number of observations in parentheses.

\begin{tabular}{ccccc}
\hline \hline Rounds & No Comm. & Binary Comm. & Token Revelation & Chat \\
\hline \multicolumn{5}{c}{$\underline{C=1}$} \\
& & & \\
$1-10$ & $37.1,12.9(70)$ & $36.3,15.0(80)$ & $47.5,6.3^{*}(80)$ & $8.3^{* * *}, 11.7(60)$ \\
$11-20$ & $45.7,14.3(70)$ & $41.3,6.3^{*}(80)$ & $40.0,11.3^{*}(80)$ & $1.7^{* * *}, 1.7^{* * *}(60)$ \\
All rounds & $41.4,13.6(140)$ & $38.8,10.6(160)$ & $43.8,8.8^{*}(160)$ & $5.0^{* * *}, 6.7^{* *}(120)$ \\
& & $\underline{C}=1.5$ & \\
$11-10$ & $46.3,6.3(80)$ & $30.0^{* *}, 6.3(80)$ & $47.5,5.0(80)$ & $45.0,3.8(80)$ \\
All rounds & $46.9,6.9(160)$ & $34.4^{* *}, 4.4(160)$ & $43.8,6.3(160)$ & $37.5^{* *}, 4.4(160)$ \\
\hline
\end{tabular}

* significant at $10 \%$; ${ }^{*}$ significant at $5 \%$; ${ }^{* *}$ significant at $1 \%$. These significance levels are for difference in proportions z-tests across the communication treatment and 'No Communication'.

Table 17: Percentage of wasteful contributions: first entry is for exactly one person contributing and second entry is for all three members in a group contributing. Number of observations in parentheses. 


\begin{tabular}{ccccc}
\hline \hline Rounds & No Comm. & Binary Comm. & Token Revelation & Chat \\
\hline \multicolumn{5}{c}{ C $=1$} \\
$1-10$ & $20.7(29)$ & $38.5^{*}(26)$ & $29.4(34)$ & $13.6(44)$ \\
$11-20$ & $15.0(20)$ & $32.1^{*}(28)$ & $16.1(31)$ & $12.1(58)$ \\
All rounds & $18.4(49)$ & $35.2^{* *}(54)$ & $23.1(65)$ & $12.8(102)$ \\
& & & & \\
& & & & \\
$1-10$ & $23.1(26)$ & $16.7(30)$ & $22.2(27)$ & $9.1(22)$ \\
$11-20$ & $6.3(16)$ & $25.0^{*}(28)$ & $21.4(14)$ & $10.0(30)$ \\
All rounds & $16.7(42)$ & $20.7(58)$ & $22.0(41)$ & $9.6(52)$ \\
\hline
\end{tabular}

* significant at $10 \% ; * *$ significant at $5 \%$; ** significant at $1 \%$. These significance levels are for difference in proportions z-tests across the communication treatment and 'No Communication'.

Table 18: Percentage of times the contributors are not the members with the lower two costs, conditional on exactly two contributing. Number of observations where exactly two contribute in parentheses. 